\title{
A sublethal ATP11A mutation associated with neurological deterioration causes aberrant phosphatidylcholine flipping in plasma membranes
}

Katsumori Segawa, ${ }^{1}$ Atsuo Kikuchi, ${ }^{2}$ Tomoyasu Noji, ${ }^{3}$ Yuki Sugiura, ${ }^{4}$ Keita Hiraga, ${ }^{3}$ Chigure Suzuki, ${ }^{5,6}$ Kazuhiro Haginoya, ${ }^{7,8}$ Yasuko Kobayashi, ${ }^{79}$ Mitsuhiro Matsunaga, ${ }^{1}$ Yuki Ochiai, ${ }^{1}$ Kyoko Yamada, ${ }^{1}$ Takuo Nishimura, ${ }^{1}$ Shinya Iwasawa, ${ }^{2}$ Wataru Shoji, ${ }^{10}$ Fuminori Sugihara, ${ }^{11}$ Kohei Nishino, ${ }^{12}$ Hidetaka Kosako, ${ }^{12}$ Masahito Ikawa, ${ }^{13}$ Yasuo Uchiyama, ${ }^{5,6}$ Makoto Suematsu, ${ }^{4}$ Hiroshi Ishikita, ${ }^{3}$ Shigeo Kure, ${ }^{2,14}$ and Shigekazu Nagata ${ }^{1,15}$

'Laboratory of Biochemistry and Immunology, World Premier International Research Center, Immunology Frontier Research Center, Osaka University, Suita, Osaka, Japan. ${ }^{2}$ Department of Pediatrics, Tohoku University School of Medicine, Sendai, Miyagi, Japan. ${ }^{3}$ Research Center for Advanced Science and Technology, The University of Tokyo, Tokyo, Japan. ${ }^{4}$ Department of Biochemistry, Keio University School of Medicine, Tokyo, Japan. ${ }^{5}$ Department of Cellular and Molecular Pharmacology and ${ }^{6}$ Department of Cellular and Neuropathology, Juntendo University Graduate School of Medicine, Tokyo, Japan. ${ }^{7}$ Department of Pediatric Neurology, Takuto Rehabilitation Center for Children, Sendai, Miyagi, Japan. ${ }^{8}$ Department of Pediatric Neurology, Miyagi Children's Hospital, Sendai, Miyagi, Japan. ${ }^{9}$ Department of Pediatrics, National Hospital Organization Sendai-Nishitaga Hospital, Sendai, Miyagi, Japan. ${ }^{10}$ Frontier Research Institute for Interdisciplinary Sciences, Tohoku University, Sendai, Miyagi, Japan. ${ }^{11}$ Central Instrumentation Laboratory, Research Institute for Microbial Diseases, Osaka University, Suita, Osaka, Japan. ${ }^{2}$ Division of Cell Signaling, Fujii Memorial Institute of Medical Sciences, Tokushima University, Tokushima, Japan. ${ }^{13}$ Department of Experimental Genome Research, Research Institute for Microbial Diseases, Osaka University, Suita, Osaka, Japan. ${ }^{14}$ Tohoku Medical Megabank Organization, Tohoku University, Sendai, Miyagi, Japan. ${ }^{15}$ Center for Infectious Disease Education and Research, Osaka University, Suita, Osaka, Japan.

ATP11A translocates phosphatidylserine (PtdSer), but not phosphatidylcholine (PtdCho), from the outer to the inner leaflet of plasma membranes, thereby maintaining the asymmetric distribution of PtdSer. Here, we detected a de novo heterozygous point mutation of ATP11A in a patient with developmental delays and neurological deterioration. Mice carrying the corresponding mutation died perinatally of neurological disorders. This mutation caused an amino acid substitution (Q84E) in the first transmembrane segment of ATP11A, and mutant ATP11A flipped PtdCho. Molecular dynamics simulations revealed that the mutation allowed PtdCho binding at the substrate entry site. Aberrant PtdCho flipping markedly decreased the concentration of PtdCho in the outer leaflet of plasma membranes, whereas sphingomyelin (SM) concentrations in the outer leaflet increased. This change in the distribution of phospholipids altered cell characteristics, including cell growth, cholesterol homeostasis, and sensitivity to sphingomyelinase. Matrix-assisted laser desorption ionization-imaging mass spectrometry (MALDI-IMS) showed a marked increase of SM levels in the brains of Q84E-knockin mouse embryos. These results provide insights into the physiological importance of the substrate specificity of plasma membrane flippases for the proper distribution of PtdCho and SM.

\section{Introduction}

Lipids have various roles in cells, including that of energy source, membrane structural component, signaling molecule, and platform for the recruitment and activation of enzymes (1). Their backbone (glycerophospholipids, sphingolipids, and cholesterol), the size of the acyl chains, and the number of double bonds vary widely. Major glycerophospholipids are phosphatidylcholine (PtdCho), phosphatidylethanolamine (PtdEtn), phosphatidylserine (PtdSer), phosphatidylinositol (PtdIns), and phosphatidic acids (PtdOH). Plasma membranes in eukaryotes comprise a lipid bilayer and are rich in PtdCho (43\% of phospholipids), PtdEtn (21\%), sphingomyelin (SM) (23\%), and PtdSer $(4 \%)(2,3)$. They also

Authorship note: KS and AK contributed equally to this work. Conflict of interest: $\mathrm{KY}$ is on a leave of absence from Chugai Pharmaceutical Co. Copyright: ( 2021, American Society for Clinical Investigation. Submitted: January 22, 2021; Accepted: August 5, 2021; Published: August 17, 2021. Reference information: J Clin Invest. 2021;131(18):e148005. https://doi.org/10.1172/JCl148005 contain a high level of cholesterol. Plasma membranes of human fibroblasts and rat hepatocytes were reported to contain cholesterol at a level of approximately $45 \mathrm{~mol} \%$ of all phospholipids $(4,5)$.

In mammalian cells, the final step in the synthesis of glycerophospholipids (PtdSer, PtdCho, and PtdIns) occurs at the cytoplasmic side of the ER or the mitochondria-associated membrane, whereas SM is synthesized at the luminal side of the Golgi apparatus $(2,3)$. After their synthesis, glycerophospholipids are transported by vesicular trafficking and lipid transfer proteins to the plasma membrane, where they are asymmetrically distributed between the lipid bilayers. PtdSer, PtdEtn, and PtdIns exclusively localize to the inner leaflet of plasma membranes, whereas most, if not all, of the PtdCho and SM are present in the outer leaflet (6, 7). The translocation of phospholipids and SM from the inner to the outer leaflet has been proposed to be mediated by floppase(s), with some ATP-binding cassette (ABC) transporters being proposed as the candidate transporters in this process (8). However, the contribution of $\mathrm{ABC}$ transporters to maintenance of the asymmetrical distribution of PtdCho and SM remains elusive. In con- 
trast, type IV-P-type (P4) ATPases are responsible for maintaining PtdSer and PtdEtn at the inner leaflet of plasma membranes (9).

P4-ATPases are membrane proteins that consist of 10 transmembrane segments and a cytoplasmic ATPase domain (10, 11). Loss-of-function mutations in 3 of the 14 P4-ATPases have been implicated in human genetic diseases, such as intrahepatic cholestasis, anemia, and neurological disorders $(8,9,12)$. The majority of P4-ATPases require CDC50A for their proper cellular localization and ATPase activity (13-15). We previously demonstrated that 2 members (ATP11A and ATP11C) of the P4-ATPase family were widely expressed in human and mouse tissues, localized in plasma membranes together with CDC50A, and specifically translocated PtdSer and PtdEtn from the outer leaflet to the inner leaflet of plasma membranes $(14,16,17)$.

In the present study, we describe a de novo heterozygous point mutation of the ATP11A gene found in a patient with global developmental delays and neurological deterioration. This mutation allowed ATP11A to bind to PtdCho at the phospholipid entry site in order to flip it. The flipping of PtdCho altered various cell characteristics, such as sensitivity to sphingomyelinase (SMase), cholesterol homeostasis, and growth rate, indicating that PtdCho needs to be asymmetrically distributed in the plasma membrane in order to maintain the integrity of mammalian cells.

\section{Results}

Clinical features of the patient. The patient was a 26-year-old male born normally at 38 weeks of gestation as a single child of healthy, nonconsanguineous Japanese parents. At birth, his weight, length, and occipitofrontal head circumference were $2186 \mathrm{~g}$ (-1.9 SD), $42.0 \mathrm{~cm}$ (-3.3 SD), and $31.5 \mathrm{~cm}$ (-1.3 SD), respectively. He developed epilepsy at 2 weeks of age, followed by a global developmental delay with mild hypothyroidism and cataracts. At the age of 2 years, he could laugh and gained gazing and eye-tracking ability; however, his overall developmental quotient was 13. He walked with a posture control walker until elementary school. He then gradually lost developmental milestones. Infectious diseases, such as aspiration pneumonia, frequently worsened his neurological deterioration. At the age of 18 years, his weight, length, and occipitofrontal head circumference were $26.0 \mathrm{~kg}$ (-3.6 SD), 140 $\mathrm{cm}(-4.6 \mathrm{SD})$, and $52.5 \mathrm{~cm}(-4.0 \mathrm{SD})$, respectively. He is currently bedridden and on mechanical ventilation. Eye movement is present without gazing or eye tracking. He has stiffness of the posterior neck and limbs and flexion contractures of the limbs without pyramidal signs. The patient also exhibits tongue fasciculation and no deep tendon reflexes. Although his white blood cell count is within the normal range $\left(6.1 \times 10^{9}\right.$ cells/L), the percentage of CD19+ B lymphocytes is significantly reduced $(1.0 \%$ [reference value: 2.9-20.1]). His serum immunoglobulin level is normal. Electroencephalography showed no prominent epileptic activity. Repeated brain MRI revealed nonprogressive, severe cerebral atrophy with ventriculomegaly, an enlarged subarachnoid space, hypomyelinating leukodystrophy, and thinning of the corpus callosum (Figure 1). A motor nerve conduction study indicated mild-to-moderate reductions in velocities of the post-tibial nerve ( $43.2 \mathrm{~m} / \mathrm{s},-1.5 \mathrm{SD})$ and median nerve $(50.0 \mathrm{~m} / \mathrm{s},-3.0 \mathrm{SD})$ and a severe decrease in compound muscle action potentials in the post-tibial nerve (1.03 $\mathrm{mV},-4.0 \mathrm{SD})$, suggesting axonal neuropathy.
Identification of the de novo heterozygous mutation of ATP11A. We performed whole-exome sequencing of DNA obtained from the patient and his parents. After filtering coding variants against allele frequencies in public and local databases (Table 1), we identified the heterozygous de novo variant c. $250 \mathrm{C}>\mathrm{G}$ in exon 3 of the ATP11A gene in the patient and confirmed this by Sanger sequencing (Figure 2A). The resulting amino acid change (Q84E) affected one of the highly conserved residues in the first transmembrane segment of ATP11A (Figure 2B). Real-time reverse transcription PCR (RT-PCR) of skin fibroblasts from the patient confirmed that the mutant allele was transcribed to mRNA as efficiently as the WT allele (Figure 2C).

To establish whether the identified heterozygous mutation of ATP11A was responsible for the patient's phenotypes, we designed a conditional knockin allele $\left(A t p 11 a^{f}\right)$ to express WT Atp11a before Cre-mediated recombination and induced expression of the Q84E mutant (c.250C $>$ G) allele after recombination (Supplemental Figure 1; supplemental material available online with this article; https://doi.org/10.1172/JCI148005DS1). Crossing of the Atp11at/WT male mice with homozygous $C A G$-Cre female mice generated few Atp11a ${ }^{Q 84 E / W T}$ mice that developed until the weaning stage (3 mutant mice among 15 mice from 3 pregnant mice). Following in vitro fertilization (IVF) or intracytoplasmic sperm injection (ICSI) with sperm from the surviving Atp11 $a^{844 / W T}$ mice, $80 \%$ of mutant mice died within 1 week of birth (number of surviving mice: 38 vs. 6 for Atp11 $a^{W T / W T}$ and Atp11a $a^{Q 84 E / W T}$ mice, respectively). We observed no apparent sex differences in the mutant mice. Atp11a ${ }^{\text {Q4E } / W T}$ mice surviving beyond the perinatal period grew slower than did WT mice (Figure 3A), and 4 of 7 died within 5 weeks of birth (Figure 3B). Before death, the Atp11a ${ }^{Q 84 E / W T}$ mice displayed neurological deficit phenotypes, including tremors (Supplemental Videos 1, 2, and 3), an abnormal gait (Figure 3C), and hind limb clasping (Figure 3D). An MRI analysis of mice at 11 weeks showed an apparent reduction in brain size $\left(421.7\right.$ vs. $\left.495.4 \mathrm{~mm}^{3}\right)$ and dilated lateral ventricles in Atp11a $a^{\mathrm{Q} 4 \mathrm{E} / W T}$ mutant mice (Figure 3E and Supplemental Figure 2). Histological analysis and electron microscopy observations revealed tissue degeneration with pyknotic or TUNEL-positive neurons in various brain regions of Atp11a ${ }^{\text {Q8E E } / W T}$ embryos and pups (Figure 3, F and G). The average number of TUNEL-positive foci in whole-brain sections of mutant mice was approximately 4 -fold higher than that in WT mice (Figure 3G). Sequencing analysis of RNA from the brains and spleens of affected mice indicated that half of the mRNA carried the c.250C $>$ G mutation (Supplemental Figure 3). To confirm that the mutant protein was expressed in vivo, we performed SDS-PAGE to separate the membrane fraction of mouse placenta that abundantly expressed ATP11A (18). Proteins of approximately $110-130 \mathrm{kDa}$ were digested with chymotrypsin and subjected to a targeted analysis by liquid chromatography tandem mass spectrometry (LC-MS/MS) (19). Figure 3H and Supplemental Figure 4 show that the Q84-containing WT ATP11A peptide (LVQLIIDTPTSPVTSGLPL) was present in the chymotryptic digests of the placenta membrane fraction of both Atp11 $a^{W T / W T}$ and Atp11a $a^{Q 84 E / W T}$ mice. In contrast, we detected the E84-containing mutant peptide (LVELIIDTPTSPVTSGLPL) only in digests of the placenta membrane fraction of Atp11a $a^{\mathrm{Q} 4 E / W T}$ mice. These results confirmed that the heterozygous point mutation of Atp11a was dominant for causing neuronal defects and lethality. 
A

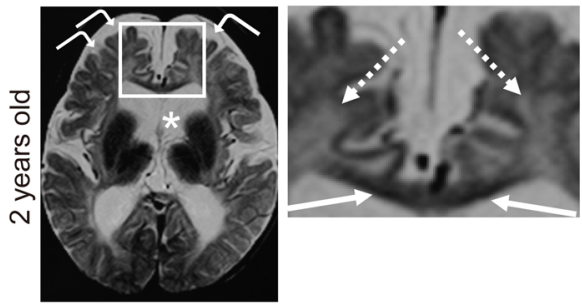

D

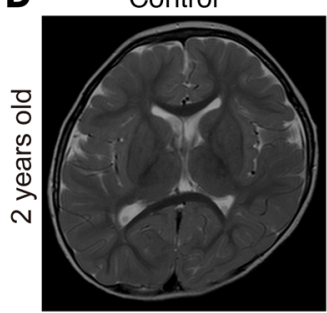

G

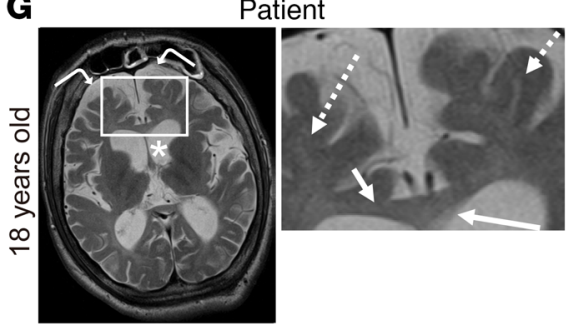

$\mathbf{K}$

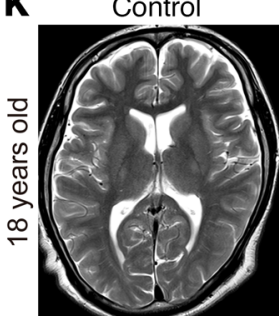

B

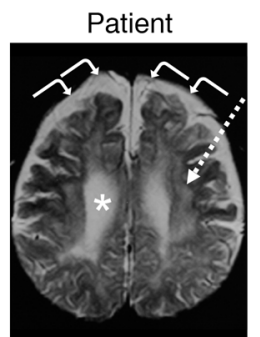

E
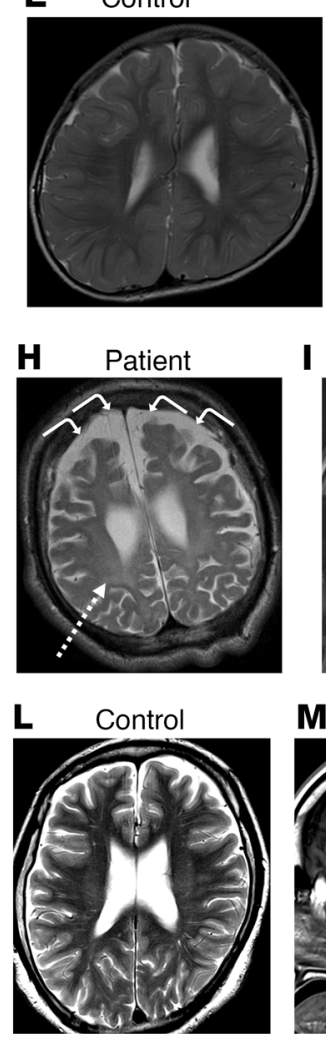

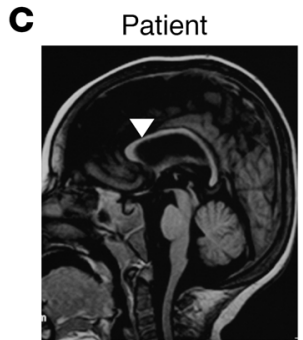

F Control

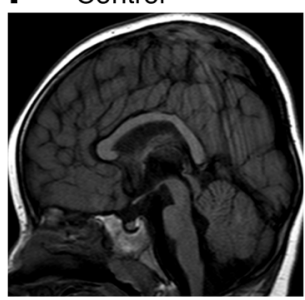

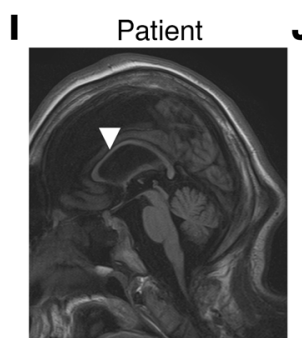

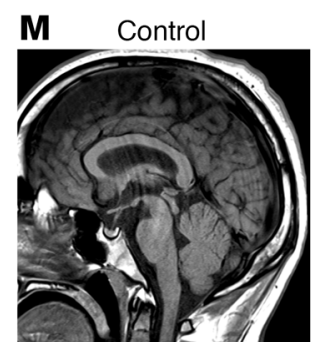

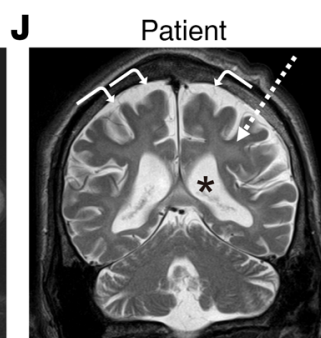

N Control

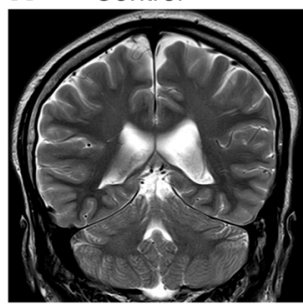

Figure 1. Brain MRI. Brain MRI of the patient (A-C and $\mathbf{G}-\mathbf{J})$ and age-matched normal controls (D-F and $\mathbf{K}-\mathbf{N})$ at 2 years $(\mathbf{A}-\mathbf{F})$ and 18 years $(\mathbf{G}-\mathbf{N})$ of age. T2-weighted axial (A, B, D, E, G, H, K, and L), T2-weighted coronal (J and $\mathbf{N}$ ), and T1-weighted sagittal (C, F, I, and $\mathbf{M})$ images are shown. The boxed areas in $\mathbf{A}$ and $\mathbf{G}$ were enlarged and are shown on the right. Cerebral atrophy with ventriculomegaly (asterisks) and an enlarged subarachnoid space (curved arrows) were observed (A, B, G, H, and J). An abnormal T2 hyperintense signal of the diffuse cerebral white matter (dashed arrows) indicated hypomyelination (A, B, G, H, and J). The patient's corpus callosum at 2 years of age showed a T2 hypointense signal (arrows, magnified field in $\mathbf{A}$ ), indicating normal myelination, while an abnormal T2 hyperintense signal in the corpus callosum (arrows, magnified field in $\mathbf{G}$ ) was observed in the patient at 18 years of age. Midline sagittal T1-weighted brain MRI demonstrated a thin corpus callosum (arrowheads in C and I). Cerebral atrophy did not appear to have progressed in the patient between the ages of 2 and 18 years.

Flipping of PtdCho by the Q84E-ATP11A mutant. Previous studies of yeast P4-ATPase (Drs2) indicated that Q84, localized to the interfacial region between the extracellular fluid and plasma membrane, functions as a substrate-selecting entry gate (20). To determine whether $\mathrm{Q} 84 \mathrm{E}$ has an altered substrate specificity, we stably expressed GFP-tagged WT or Q84E- human ATP11A (hATP11A) in the Atp11a $a^{-1}$ Atp11 $c^{-/-}$W3 mouse T-lymphoma cell line (double-knockout [DKO]) (17) together with hCDC50A. Both WT and Q84E-hATP11A were present at similar levels in plasma membranes (Figure 4A). We assessed flippase activity in the plasma membrane using a fluorescent nitro-benzoxadiazole-conjugated (NBD-conjugated) phospholipid analog (see Methods). As reported previously (14), WT ATP11A specifically promoted the incorpo- ration of NBD-PtdSer (NBD-PS) and NBD-PtdEtn (NBD-PE), but not NBD-PtdCho (NBD-PC), NBD-SM, or NBD-galactsylceramide (NBD-GalCer) (Figure 4B). Q84E exhibited flippase activity against NBD-PS and NBD-PE, but not against NBD-SM or NBD-GalCer. However, the mutant exhibited flippase activity against NBD-PC. Some yeast P4-ATPases (Dnflp and Dnf2p) have been shown to transport lyso-PtdEtn and lyso-PtdCho (21). However, neither Q84E nor WT hATP11A internalized NBD-lyso-PtdCho.

When the Q84E mutant was introduced into WT W3 cells, the transformants showed enhanced flippase activity toward PtdSer and PtdEtn. They also incorporated PtdCho as efficiently as did DKO-Q84E cells (Figure 4C and Supplemental Figure 5A), indicating that the WT alleles (Atp11a and Atp11c) of plasma mem- 


\section{Table 1. Variant filtering strategy}

\section{Filters}

\begin{tabular}{|c|c|}
\hline Total number of variants detected & 881,952 \\
\hline Filtering for low-quality variants & 809,602 \\
\hline Keep exonic variants & 22,172 \\
\hline Keep nonsynonymous and splice site variants & 10,538 \\
\hline Filtering for variants in segmental duplications & 9259 \\
\hline $\begin{array}{l}\text { MAF }<0.005 \text { in databases }{ }^{A} \\
\text { (for autosomal-recessive and X-linked inheritance) }\end{array}$ & 260 \\
\hline Absent in databases ${ }^{A}$ (for de novo inheritance) & 63 \\
\hline De novo inheritance & 1 gene $^{B}$ \\
\hline Autosomal-recessive inheritance & 1 gene $^{\mathrm{C}}$ \\
\hline X-linked inheritance & 2 genes $^{D}$ \\
\hline Keep variants with CADD Phred score $>20^{\mathrm{E}}$ & ATP11A \\
\hline Sanger sequencing validation & ATP11A \\
\hline
\end{tabular}

MAF, minor allele frequency. ${ }^{A} 1000 g 2015 a u g \_a l l, ~ e s p 6500$ siv2_all,

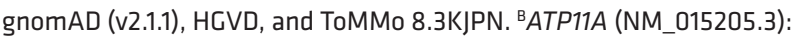
c.250C>G, p.Gln84Glu. ' $5 Y C E 1$ (NM_001143764.3): c.[585delC];[941G>A], p.[(Leu196Serfs*14)];[(Gly314Glu)]. ' MAGEB18 (NM_173699.4): c.965C>A, p.(Ala322Asp), XPNPEP2 (NM_003399.4): c.1181A>G, p.(Glu394Gly). EA CADD Phred-scaled score of 20 represents the top $1 \%$ of the most deleterious variants.

brane flippases (14) did not inhibit the PtdCho-flipping activity of Q84E-ATP11A. The PtdSer and PtdCho flippase activity induced by the Q84E mutant of ATP11A were inhibited by orthovanadate, an inhibitor of P4-ATPases (Supplemental Figure 5B), suggesting that PtdCho-flipping activity was due to the mutant ATP11A. An examination of the ATPase activity of purified recombinant WT and Q84E-ATP11A proteins indicated that palmitoyl-oleoylglycerophosphoserine (POPS) and palmitoyl-oleoyl-glycerophosphoethanolamine (POPE), but not palmitoyl-oleoyl-glycerophosphocholine (POPC) or SM, activated the ATPase activity of WT ATP11A as previously reported (refs. 13, 14 and Figure 4D). POPS and POPE activated the ATPase activity of Q84E-ATP11A. However, in contrast to WT ATP11A, the ATPase activity of Q84EATP11A was activated by POPC with approximately $20 \%$ of the efficiency of POPS (Figure 4D).

To clarify whether the replacement of Gln by Glu, but not by other amino acids, is essential for PtdCho flipping, Q84 of ATP11A was replaced by Ala, Asp, or Asn and expressed in DKO cells (Supplemental Figure 5, C and D). As shown in Figure 4E, all mutants exhibited PtdSer-flipping activity to varying degrees. In contrast, PtdCho-flipping activity was only observed with Q84E, indicating that the replacement of Q84 by Glu specifically allowed ATP11A to translocate PtdCho.

Molecular dynamics simulations for phospholipid flipping. Among human P4-ATPases, the members of 2 subfamilies (class 1a: ATP8A1 and ATP8A2, class 6: ATP11A and ATP11C) have been shown to have PtdSer-flipping activity at recycling endosomes (22) or plasma membranes $(14,16)$. The shared identities of the amino acid sequence of hATP11A with those of hATP8A1, hATP8A2, and hATP11C were 37\%, 37\%, and 66\%, respectively. The tertiary structures of hATP8A1 and hATP11C indicated that PtdSer was translocated via a cleft that formed between transmem- brane segments $(10,11,23)$. The conservation of the amino acid sequence of transmembrane segments was better between hATP11A and hATP11C (shared identity of 79\%) than between hATP11A and hATP8A1 or ATP8A2 (shared identity of $42 \%$ or $39 \%$, respectively). Since hATP11C and hATP8A2, but not hATP8A1, localized to the plasma membrane (14), we examined the effects of the Q84E mutation on flippase activity with hATP8A2 and hATP11C. We found that Q107 of hATP8A2 or Q79 of hATP11C, corresponding to Q84 of hATP11A (Supplemental Figure 6A), was mutated to Glu or Ala and expressed in DKO cells. WT hATP8A2, hATP11C, and their mutants localized to the plasma membrane (Supplemental Figure 6B). WT hATP8A2 and hATP11C translocated NBD-PS, but not NBD-PC (Figure 5, A-D). On the other hand, PtdSer flippase activity was reduced by approximately $40 \%$ and 90\% in the Q79E-mutant hATP11C and Q107E-mutant hATP8A2, respectively (Figure 5, A and $\mathrm{B}$ ). We did not observe this decline in Ala mutants (Q79A-hATP11C and Q107A-hATP8A2), suggesting that Glu at this position interfered with PtdSer flipping. We detected PtdCho flippase activity in cells expressing the Q79E mutant of hATP11C, but not Q107E-hATP8A2 (Figure 5, C and D). As with hATP11A, we did not observe PtdCho-flipping activity with the Q79A mutant of hATP11C. These results suggest that hATP11C, but not hATP8A2, used a mechanism similar to that of hATP11A to transport phospholipids.

We conducted molecular dynamics (MD) simulations using the structure of hATP11C, hATP8A1, and their mutants $(10,11$, 23). WT hATP11C interacted with PtdSer by forming $\mathrm{H}$ - bonds with Q79, T86, T90, S91, and N352 (Figure 5, E and F, and Supplemental Figure 7) at the entry site of the longitudinal groove (11), resulting in high-affinity binding of PtdSer to hATP11C (approximately $-25 \mathrm{kcal} / \mathrm{mol})$. Similarly, PtdSer interacted with hATP8A1 via $\mathrm{H}$ bonds with Q88, T100, T101, N352, and N353 (binding affinity of $-28 \mathrm{kcal} / \mathrm{mol}$ ). The Q79E mutation of ATP11C and the Q88E mutation of hATP8A1 reduced the number of $\mathrm{H}$ bonds (Figure 5, E and F, and Supplemental Figure 7), decreasing the binding affinity of PtdSer by approximately $60 \%$. In contrast, the Ala mutation in these residues did not reduce binding affinity to PtdSer (Q79A-hATP11C and Q88A-hATP8A1; Figure 5, E and F, and Supplemental Figure 7). These results were consistent with the observed flippase activity (Figure 5, A and B). We also investigated the PtdEtn flippase activity of WT ATP11C and its mutants and conducted MD simulations (Supplemental Figure 8 and Supplemental Table 1). The PtdEtn flippase activity of Q79E-hATP11C mutants was 2-fold higher than that of WT hATP11C. The binding affinity of PtdEtn to the Q79E-hATP11C mutant was 5- to 10-fold greater than that to the WT hATP11C or the Q79A-hATP11C mutant, explaining the increase in the PtdEtn flippase activity of the Q79E-hATP11C mutant.

Consistent with the results showing that Q79E-hATP11C, but neither WT ATP11C nor the Q79A-ATP11C mutant, flipped PtdCho, our MD simulations revealed that only the Q79E mutation of hATP11C formed a salt bridge between the choline moiety and E79, resulting in 5-fold stronger binding affinity (from $0.7-3.6$ $\mathrm{kcal} / \mathrm{mol}$; Figure $5 \mathrm{G}$ and Supplemental Figure 9A). In contrast to hATP11C, the carboxyl residue of E88 of hATP8A1 engaged in electrostatic interactions with the nitrogen of peptide bonds between T100 and T101 and between T96 and G97 (Figure 5H 
A

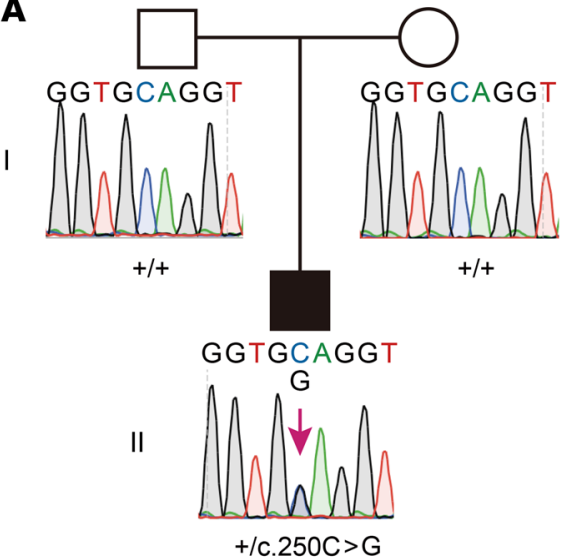

C

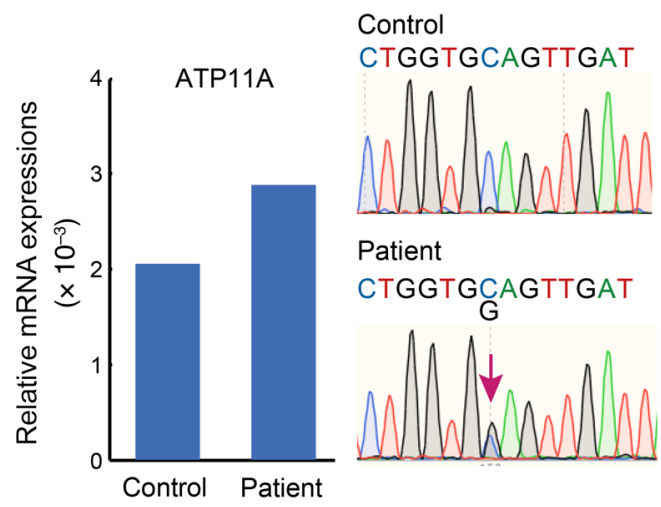

Figure 2. Identification of the Q84E mutation. (A) DNA sequences for the patient and his parents. (B) Structure of ATP11A with Q84E highlighted. Transmembrane (TM) segments are numbered. At the bottom, the amino acid sequences of ATP11A from various species are aligned. TM regions are highlighted in green and Q84 in red. Conserved residues are indicated by an asterisk. (C) Left: ATP11A mRNA levels in skin fibroblasts from a healthy individual (control) and the patient. mRNA levels are expressed relative to those of the ribosomal protein 36B4. Right: cDNA sequences for ATP11A mRNA in skin fibroblasts. The heterozygous mutation is indicated by an arrow.

B

TM1

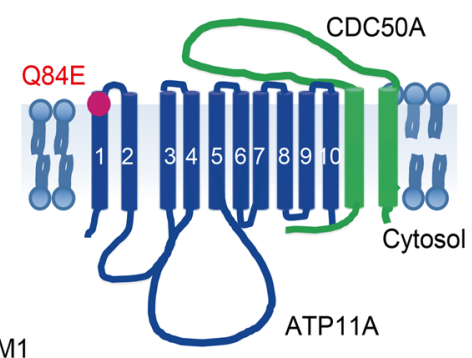

TM2

HUMan IPKNLFEOFRRVANFYFLIIFLVOLIID-TPTSPVTSGLPLFFVITVTAIKOGYEDWL

Chicken IPKNLFEQFRRIANFYFLIIFLVQLIID-TPTSPVTSGLPLLFVITVTAIKOGYEDWL

Fugu LPKNLFEQFRRIANFYFL I IFLVQVIVD-TPTSPVTSGLPLFFVITVTAIKQGYEDWL

Xenopus IPKNLFEQFRRIANFYFLI IFLIQAIVD-TPTSPVTSGLPLFFVITVTAIKQGYEDWL

Arabid. LPKNLWEQFSRFMNQYFLLIACLOLWSLITPVNPASTWGPLIFIFAVSASKEAWDDYH

Yeast LPKFLFQEFSKYANLFFLCTSAIQQVPHVSPTNRYTTIGTLLVVLIVSAMKECIEDIK

and Supplemental Figure 9B). The lack of a salt bridge between E88 and PtdCho reduced the affinity of PtdCho for Q88E-hATP8A1, supporting the lack of PtdCho-flipping activity in the Q107E mutant of hATP8A2 (Figure 5D).

Effects of PtdCho flipping on the plasma membrane. We then assessed subcellular phospholipid dynamics using TopFluortagged PtdSer (TopFluor-PS) and PtdCho (TopFluor-PC), which strongly associate with biological membranes (24). DKO and its transformants expressing WT or Q84E-hATP11A were loaded with TopFluor phospholipids and allowed to flip at $20^{\circ} \mathrm{C}$, TopFluor-PS signals were observed in plasma membranes (Figure 6A). TopFluor-PC also remained in the plasma membranes of DKO cells and DKO cells expressing WT hATP11A. In contrast, in DKO expressing Q84E, a large amount of TopFluor-PC was intracellularly distributed and merged with ER-tracker (Figure 6A). SM-loaded methyl- $\alpha$-cyclodextrin $(\mathrm{M} \alpha \mathrm{CD})$ replaces phospholipids from the outer leaflet of plasma membranes with SM (25). Accordingly, flow cytometry showed that the treatment with SM-loaded M $\alpha \mathrm{CD}$ markedly reduced TopFluor-PC fluorescence in DKO and DKO WT ATP11A cells, confirming its localization to the outer leaflet of plasma membranes (Figure 6B). In contrast, the same treatment markedly increased TopFluor-PC fluorescence in Q84E-hATP11A-expressing cells. Microscopic observations confirmed that the treatment with SM-M $\alpha \mathrm{CD}$ removed the majority of TopFluor-PC from control cells (DKO and DKO WT ATP11A), while it was strongly detected intracellularly in Q84E cells (Figure 6C).
A change in the intracellular phospholipid composition often modulates lipogenesis (26). A microarray analysis of gene expression indicated that the sphingomyelin synthase 1 (Sgms1) gene was upregulated by 10 - to 25 -fold in cells expressing the Q84E mutant (Table 2), which was confirmed by real-time RT-PCR (Figure 6D). We also detected the upregulation of Sgms1 mRNA in Q84Eexpressing WT W3 cells (Figure 6D). Western blotting with antiSGMS1 mAb showed a marked increase in the SGMS1 protein in DKO-W3 and WT W3 cells transformed with Q84E-hATP11A, but not WT hATP11A (Figure 6E). SGMS1 in the Golgi catalyzes the synthesis of SM by transferring the phosphocholine of PtdCho to ceramide (27). LC-MS/MS analysis indicated that the composition of SM species, but not other phospholipids, was approximately 2-fold higher in Q84E cells than in other control cells (parental DKO and DKO cells expressing WT hATP11A; Figure 6F, and Supplemental Figure 10).

Elevated SM at the surface of Q84E-mutant ATP11A-expressing cells. Given the elevated amounts of SM in Q84E cells, we postulated that SM replaced PtdCho to compensate for that removed by Q84E-ATP11A at the outer leaflet of plasma membranes. We observed that the staining intensity of nontoxic lysenin (NT-Lys), which specifically binds to SM-rich domains on the cell surface (28), was 6- to 8-fold higher in Q84E-expressing DKO cells than in cells expressing WT ATP11A (Figure 7A). We then metabolically labeled cellular PtdCho and SM by culturing cells for 40 hours in the presence of methyl ${ }^{-14} \mathrm{C}$-choline. Thin-layer chromatography (TLC) analysis of total cellular lipids showed an approximately 
A

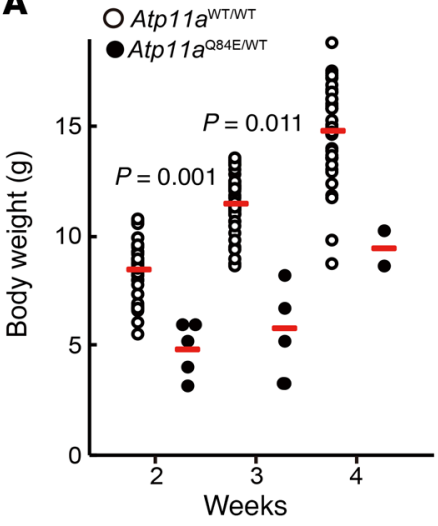

E
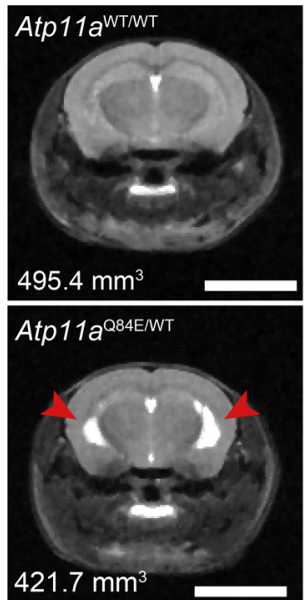

B

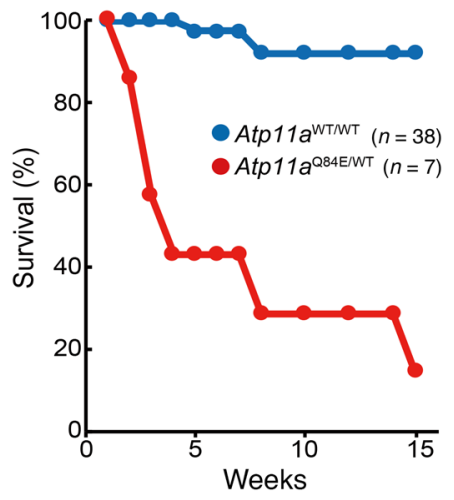

C

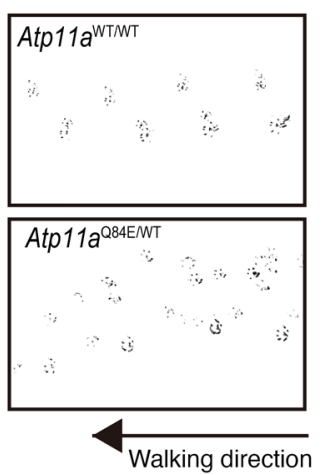

G

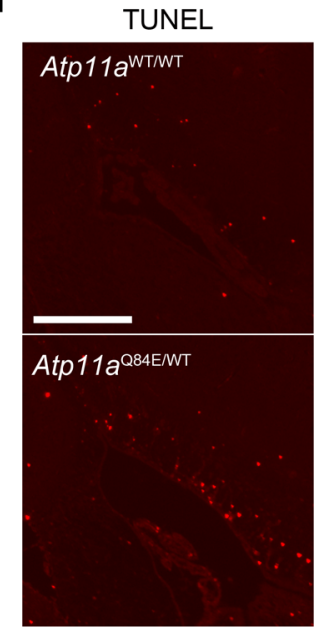

D

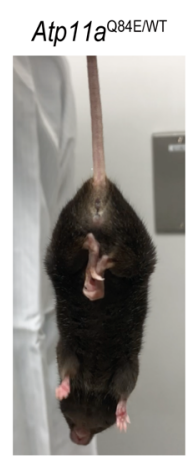

$\mathbf{F}$
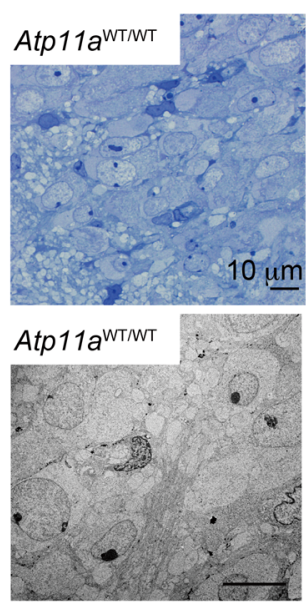
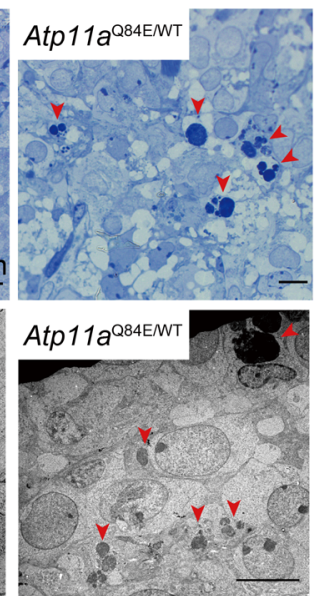

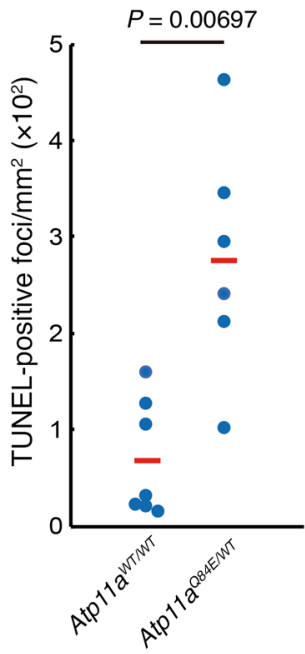

H

LVQ ${ }^{84}$ LIIDTPTSPVTSGLPL

LVE ${ }^{84}$ LIIDTPTSPVTSGLPL
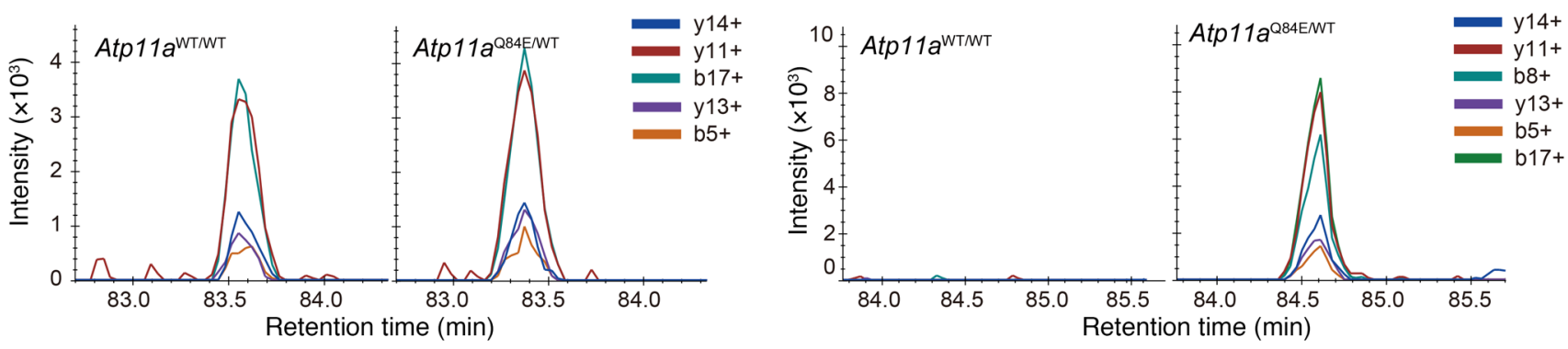

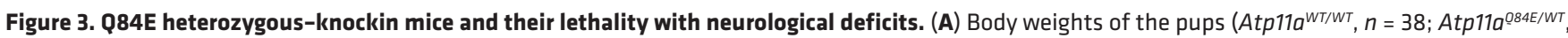
$n=5$ ). (B) Percentage of surviving pups (Atp11a ${ }^{W T / W T}, n=38$; Atp11a ${ }^{084 E / W T}, n=7$ ). (C) Gait analysis by the footprint test. After coating the paws with ink, 17-week-old female Atp11a ${ }^{084 E / W T}$ and WT littermate mice were allowed to walk. (D) Clasping of the hind limbs during tail suspension by a 20-week-old Atp11 $a^{084 E / W T}$ female mouse, a sign of a neurological defect. The same mutant mouse is shown in Supplemental Video 3. (E) Midaxial T2-weighted MRI of 11-week-old female WT and Atp11a ${ }^{084 E / W T}$ littermate mice. Red arrowheads indicate the dilated lateral ventricle. The calculated brain volume is shown. Scale bars: $5 \mathrm{~mm}$. (F) Sections of the medulla oblongata from E14 embryos were stained with toluidine blue or observed by electron microscopy. Arrowheads indicate degenerated neurons with pyknotic nuclei. Scale bars: $10 \mu \mathrm{m}$. (G) TUNEL-stained brain sections around the ventricle of 2-week-old male Atp11a ${ }^{W T / W T}$ and Atp11a ${ }^{084 E / W T}$ neonates. Scale bar: $300 \mu \mathrm{m}$. TUNEL-positive foci of 6-7 sections from different parts of the entire brain were computationally counted and are represented as TUNEL-positive foci per $\mathrm{mm}^{2} . P=0.00697$, by Student's $t$ test. (H) Presence of Q84E-containing peptide in mouse Atp11 $a^{084 E / W T}$ placenta. Proteins with a MW of 100-130 kDa in the E15 placenta membrane fraction of female Atp11a $a^{\text {WT/WT }}$ and Atp11a $a^{084 E / W T}$ mice were digested with chymotrypsin. ATP11A peptides containing Q84 (left) and E84 (right) were analyzed by PRM by using the data in Supplemental Figure 4. Extracted ion chromatograms of $\mathrm{N}$-terminal b-ions (b5+, b8+, and b17+) and C-terminal y-ions (y11+, y13+, and y14+) are shown.

2-fold higher concentration of SM in Q84E cells than in other control cells (DKO and DKO WT ATP11A) (Figure 7B), confirming the results obtained by LC-MS/MS (Figure 6F). Lipids in the outer leaflet of plasma membranes were then extracted by SM-M $\alpha \mathrm{CD}$ and analyzed by TLC. We found that the quantity of ${ }^{14} \mathrm{C}$-PtdCho was 4- to 5-fold lower in Q84E cells than in other cells, whereas the content of SM was approximately 4 -fold higher, increasing the ratio of SM to PtdCho by approximately 20-fold (Figure 7B). This 
A
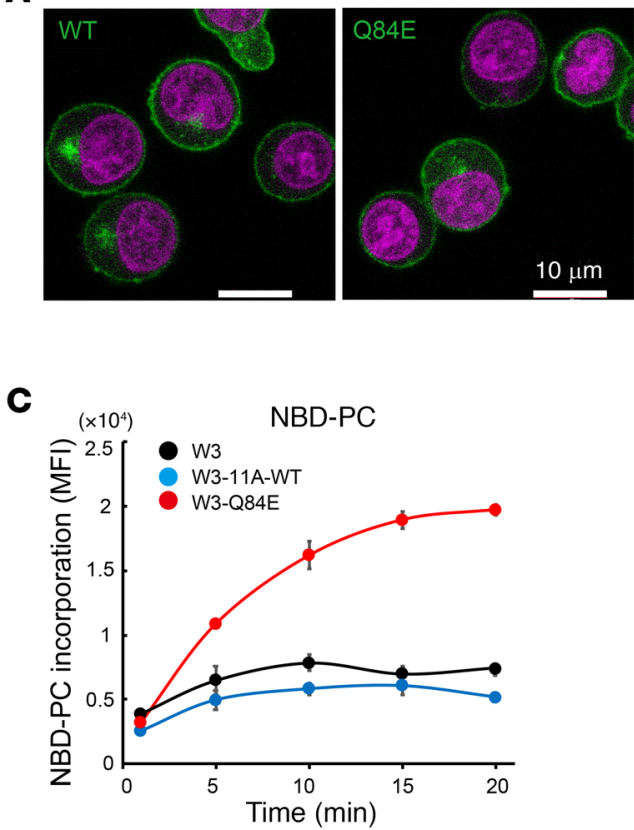

D
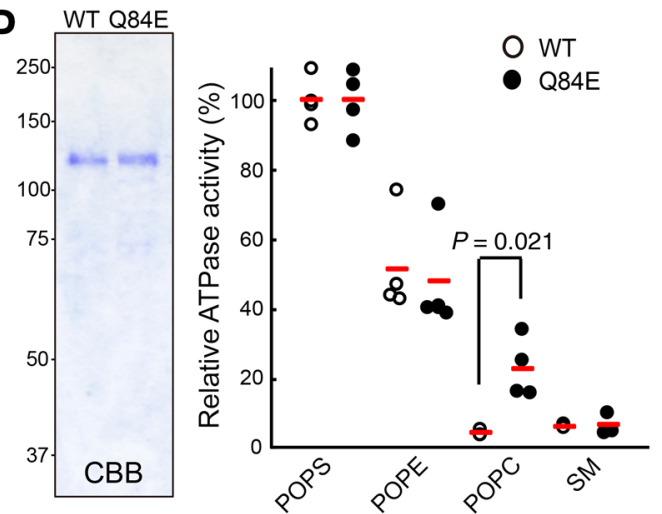

B
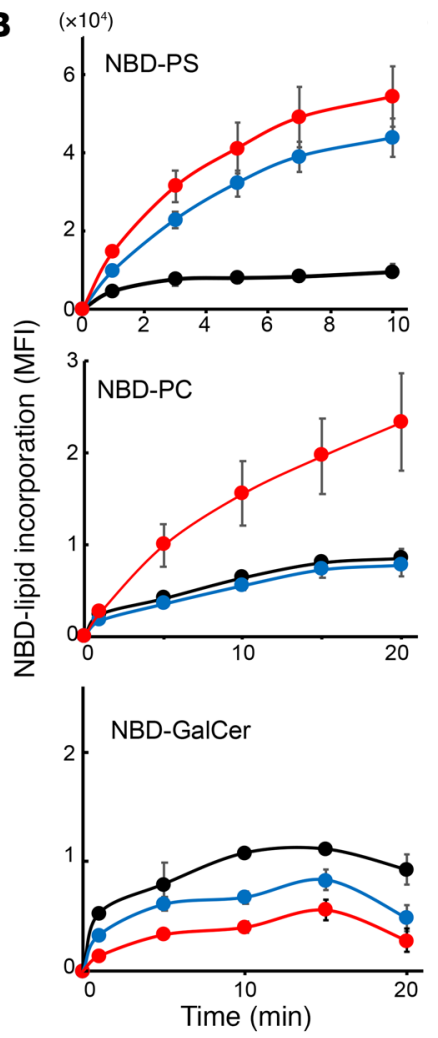

$\left(\times 10^{4}\right)$

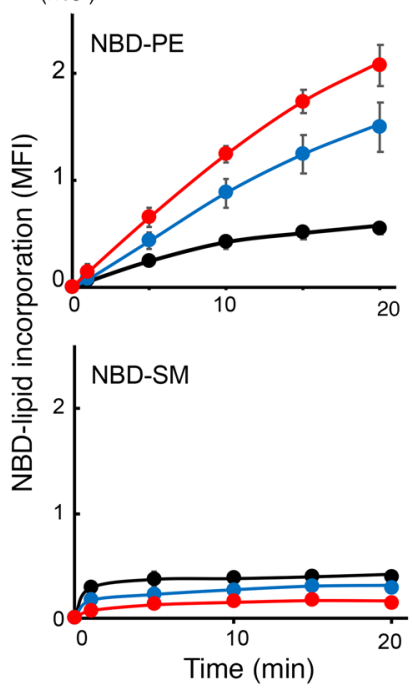

DKO

DKO-11A-WT

DKO-Q84E

E
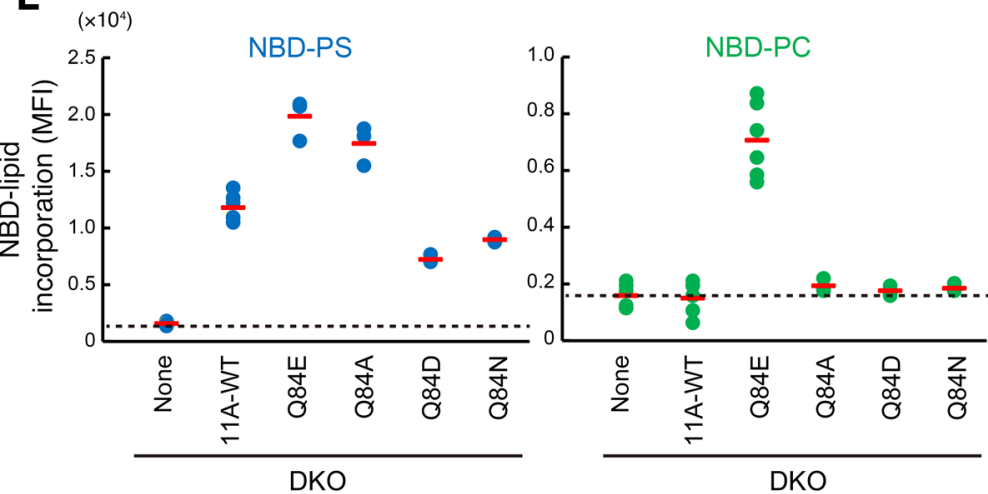

Figure 4. PtdCho-flipping activity of the Q84E mutant. (A) Membrane localization of Q84E-ATP11A. GFP (green); DRAQ5 (magenta). Scale bars: $10 \mu \mathrm{m}$. (B and C) DKO (B) or W3 (C) cells expressing WT or Q84E-ATP11A were incubated with the indicated phospholipids, and incorporated fluorescence was plotted $(n=3)$, with the SD shown. (D) SDS-PACE of WT and Q84E-ATP11A (180 ng protein). Proteins were stained with CBB. ATPase activity with the indicated phospholipids is shown as a percentage of POPS-activated ATPase $(n=4)$ with mean values (bars). $P=0.021$, by Student's $t$ test. (E) DKO and its transformants expressing the indicated mutant ATP11A were incubated with NBD-PS or NBD-PC, and the MFI is plotted $(n=3-6)$, with the average value shown.

marked increase in SM on the cell surface substantially altered cellular sensitivity to SMase. Similar to other mammalian cells, such as human proximal tubule cells (29), the treatment of DKO or DKO WT hATP11A cells with 2 units/mL SMase for 5 minutes did not induce plasma membrane rupture leading to lactate dehydrogenase (LDH) release (Figure 7C). However, a 10-fold lower concentration of SMase killed the majority of Q84E cells within 5 minutes, causing the release of LDH. WT W3 cells expressing Q84E-ATP11A showed the same characteristics as the DKO transformants (accumulation of SM on the cell surface and increased sensitivity toward SMase; Supplemental Figure 11). Collectively, these results indicate that the concentration of PtdCho marked- ly decreased in the outer leaflet of plasma membranes in Q84EhATP11A-expressing cells, while that of SM increased.

Based on its ability to bind to perfringolysin O (PFO), cholesterol in the plasma membrane is divided into "accessible" and "inaccessible" pools, with that in the accessible pool moving to the ER for esterification and storage $(4,30)$. Since cholesterol becomes "inaccessible" after binding to SM (4), the large quantity of SM in the outer leaflet of the Q84E cells may alter the distribution of cholesterol. As shown in Figure 7D, mCherry-tagged D4, a cholesterol-binding domain of PFO (31), bound to Q84E-expressing cells with approximately $50 \%$ of the efficiency of other control cells (DKO and DKO WT hATP11A). We confirmed the ability of the Q84E mutant to 

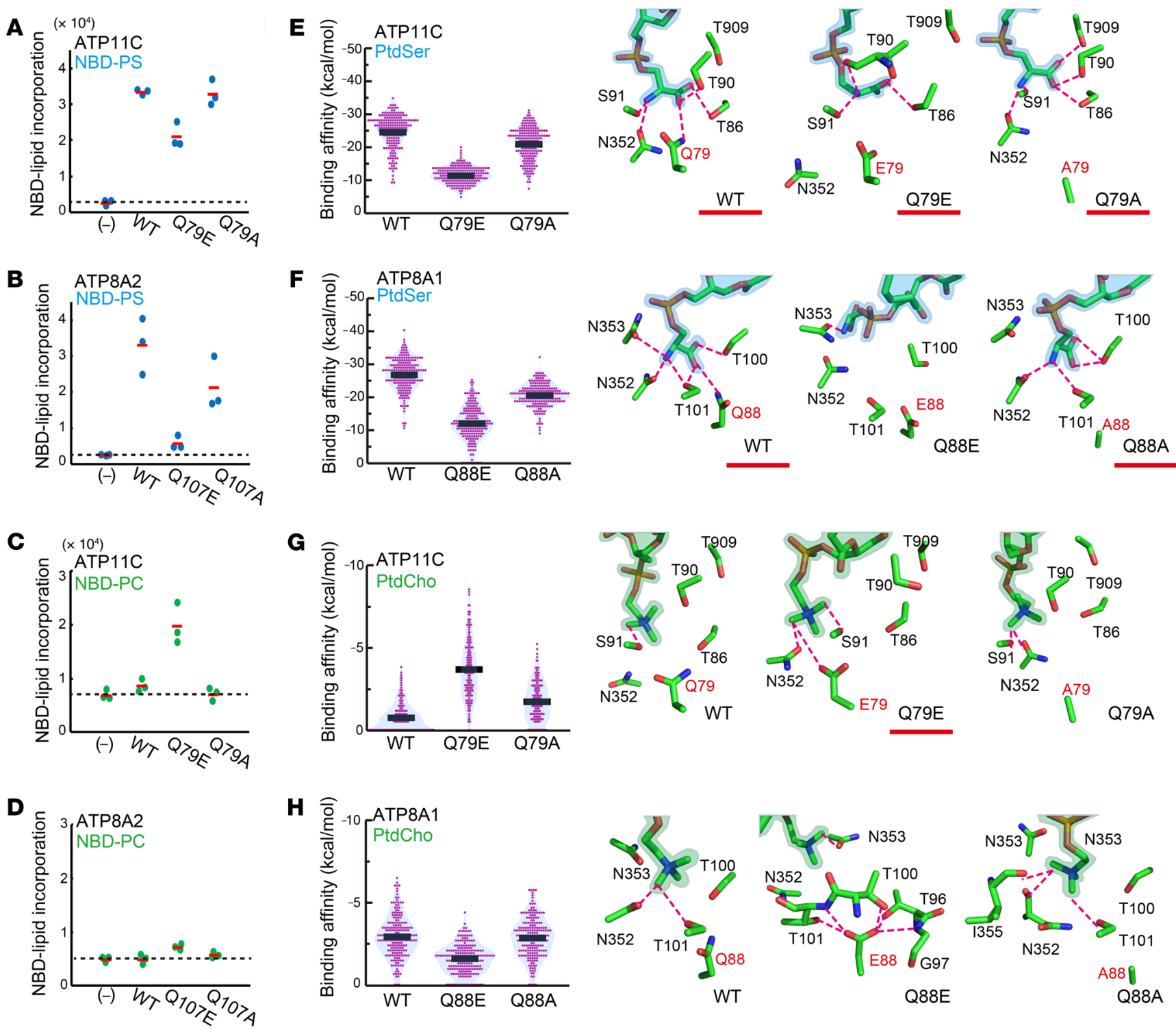

Figure 5. MD simulation. (A-D) Flippase activity. DKO cells expressing the WT or indicated mutant of hATP11C (A and C) or hATP8A2 (B and D) were incubated with NBD-PS (A and B) or NBD-PC (C and $\mathbf{D})$, and the MFI is plotted, with red bars indicating the average value. (E-H) MD analysis of the flipping of PtdSer ( $\mathbf{E}$ and $\mathbf{F}$ ) or PtdCho (G and $\mathbf{H}$ ) by the WT and indicated mutant of ATP11C (E and $\mathbf{G}$ ) or ATP8A1 (F and $\mathbf{H}$ ) was simulated as described in Methods. Calculated binding energies $(\mathrm{kcal} / \mathrm{mol})$ are shown in the violin plot; black bars indicate the average value $(n=200$ structures). Right panels show typical geometries obtained with the indicated molecules for PtdSer and PtdCho. PtdSer and PtdCho are shaded in pale blue and pale green, respectively. Dotted lines indicate electrostatic interactions. Red, oxygen; blue, nitrogen; green, amino acid carbon; gray, hydrogen; orange, phosphorous carbon. Molecules exhibiting flippase activity are underlined in red.

reduce accessible cholesterol on the cell surface in WT W3 cells (Supplemental Figure 11C). Consistent with the reductions in accessible cholesterol, cholesteryl ester levels were more than $70 \%$ lower in Q84E-expressing cells (Figure 7E). In contrast, the expression levels of SREBP-regulated genes, such as 3-hydroxy-3-methylgrutarylcoenzyme A reductase ( $\mathrm{Hmgcr}$ ), low-density lipoprotein receptor ( $L d l r)$, and fatty acid synthase (Fasn), were not affected by the Q84E mutation of ATP11A (Table 2). These results suggest that the expression of Q84E-hATP11A did not change free cholesterol levels in the ER membrane. Q84E cells showed markedly lower proliferation rates than did DKO WT ATP11A (doubling time; 12.6 vs. 9.6 hours) (Figure 7F), and were also smaller than control cells (Figure 7G).
We then assessed SM levels in E16 embryos using matrixassisted laser desorption ionization-imaging mass spectrometry (MALDI-IMS) (32). As shown in Figure 8A, the levels of $\mathrm{N}$-stearoyl-D-erythro-sphingosylphosphorylcholine [SM (d18:1/18:0)], $N$-oleoyl-D-erythro-sphingosylphosphorylcholine [SM (d18:1/18:1)], and $N$-palmitoyl-D-erythro-sphingosylphosphorylcholine [SM (d18:1/16:0)] in various regions, particularly the brain and lungs, were higher in Atp11a $a^{84 E / W T}$ than in Atp11a ${ }^{W T / W T}$ embryos. PtdCho and ceramide, substrates for the synthesis of SM, were reduced in the lungs of Atp11a $a^{Q 84 / W T}$ mice (Figure 8B), in which SM levels increased. Trophoblasts in the mouse placenta express a high level of ATP11A (18). We found SM (d18:1/16:0) levels in the placental labyrinth to 
A

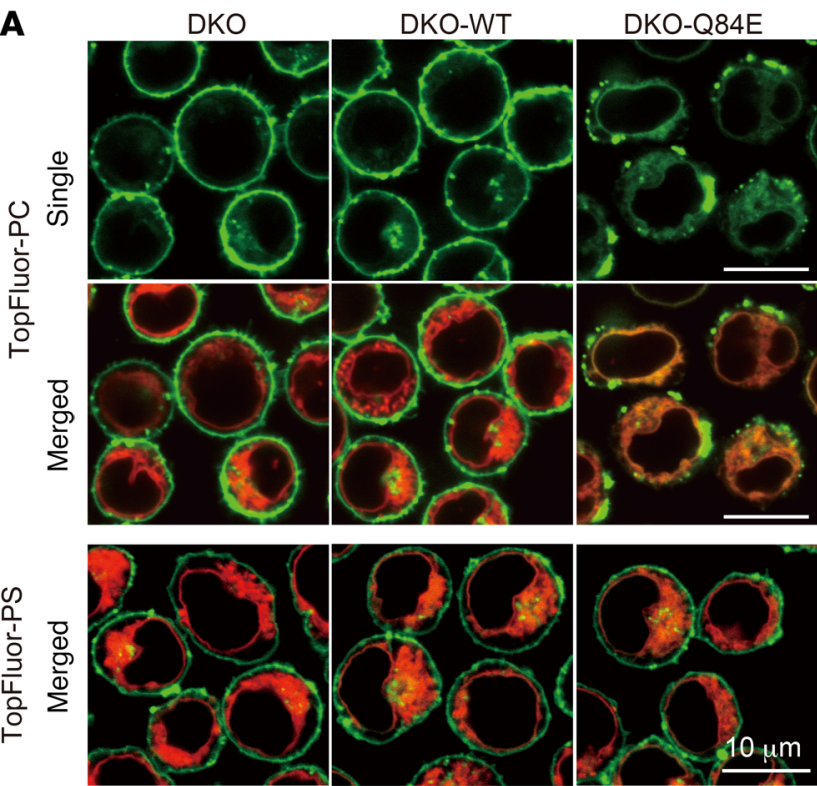

B
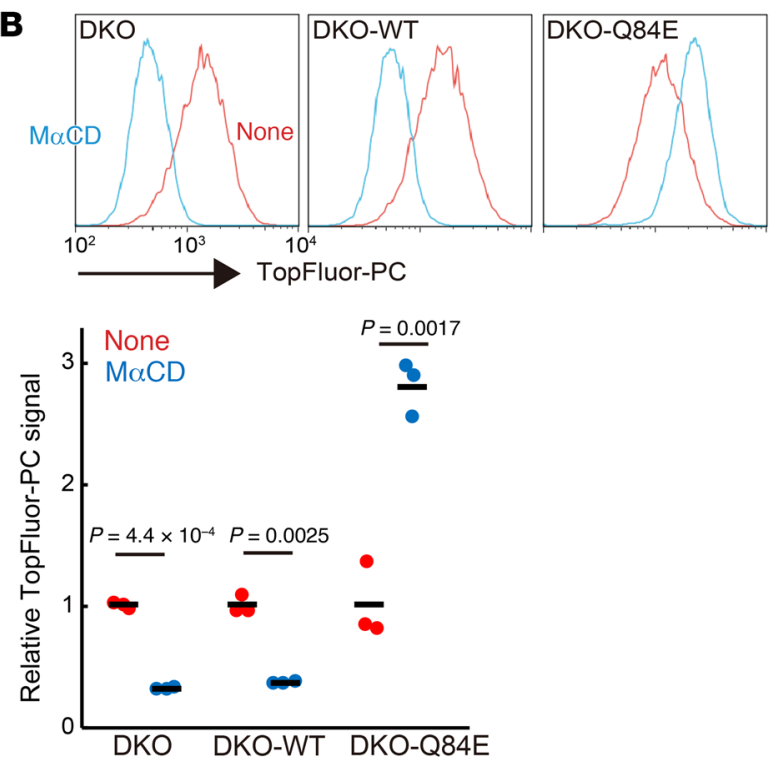

C

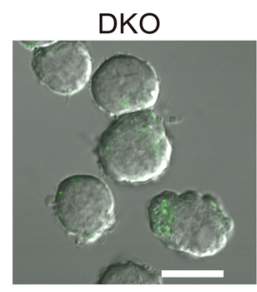

D

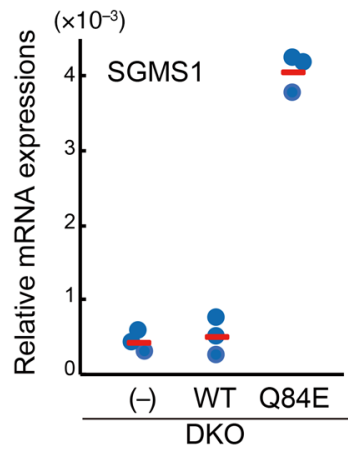

$\left(\times 10^{-3}\right)$

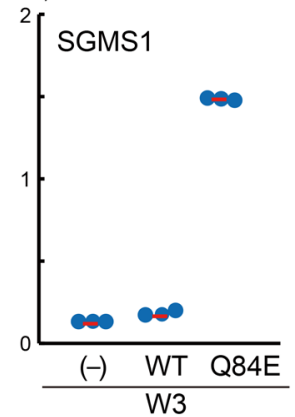

E

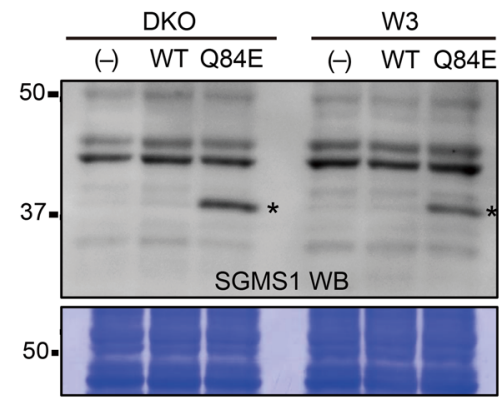

$\mathbf{F}$

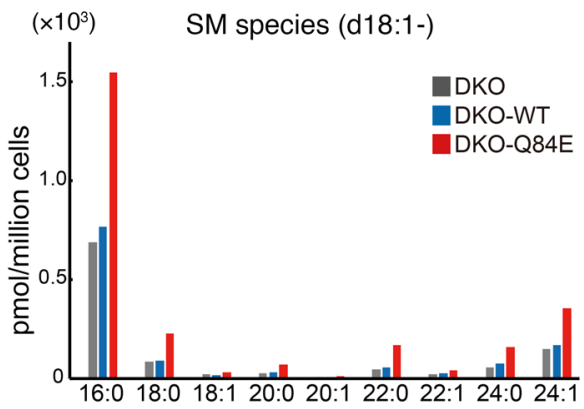

Figure 6. Increase in SM in the plasma membrane by Q84E-ATP11A. (A) Cells were loaded with TopFluor-PC or TopFluor-PS (green), incubated at 20 ${ }^{\circ} \mathrm{C}$, and observed by fluorescence microscopy in the presence of ER tracker (red). Scale bars: $10 \mu \mathrm{m}$. (B) Cells were loaded with TopFluor-PC (red), treated with $\mathrm{SM}-\mathrm{M} \alpha \mathrm{CD}$ (blue), and analyzed by flow cytometry. Experiments were performed 3 times, and TopFluor-PC signals (MFI) were normalized to signals in cells that were not treated with SM-M $\alpha$ D and plotted. $P=4.4 \times 10^{-4}$ (DKO); $P=0.0025$ (DKO WT); $P=0.0017$ (DKO-Q84E), by Student's $t$ test. (C) Cells were loaded with TopFluor-PC (green), treated with SM-M $\alpha C D$, and observed by fluorescence microscopy. Scale bars: $10 \mu \mathrm{m}$. (D and E) SCMS1 mRNA and protein levels in DKO or W3 cells expressing WT hATP11A or Q84E-hATP11A were assessed by (D) real-time RT-PCR $(n=3)$, relative to the expression of 36B4, or (E) Western blotting (WB) of total cell lysates with anti-SGMS1 mAb. The membrane used for Western blotting was stained with CBB. The asterisks indicate SCMS1. (F) The concentration of SM species in the indicated cells was assessed by LC-MS/MS.

be higher in Atp11a $a^{\text {Q8EE/WT }}$ than in Atp11a ${ }^{W T / W T}$ embryos (Figure 8C). These results suggest that the Q84E mutation of ATP11A caused the flipping of PtdCho at plasma membranes and the upregulation of SM synthesis in these tissues.

\section{Discussion}

By analyzing whole-exome sequencing data from unaffected parents-affected child trios (33) followed by biochemical and cell biological analyses, we identified a de novo mutation of the ATP11A gene in a patient with severe developmental disorders and progressive neurological decline. The same heterozygous mutation in mice recapitulated the phenotypes (growth retardation, reduction of the brain size, and ataxia) of the patient, confirming it as the dominant mutation responsible for the symptoms. ATP11A is present in the plasma membrane and exclusively translocates PtdSer and PtdEtn, but not PtdCho, from the outer 


\section{Table 2. Microarray analysis}

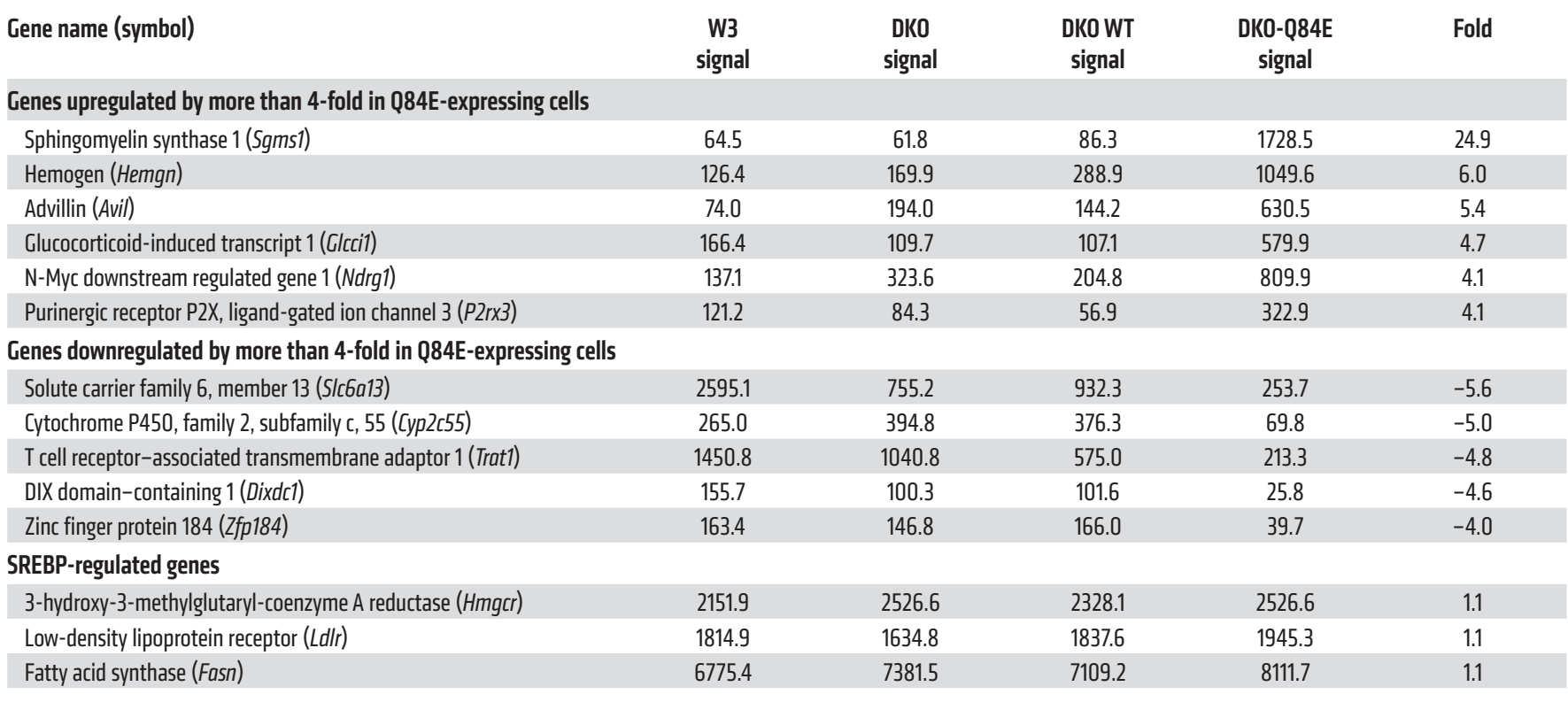

Gene expression in W3, DKO, and DKO cells expressing WT or Q84E-mutant ATP11A was determined by microarray analysis. Genes up- or downregulated by more than 4-fold in Q84E-expressing cells compared with other cells are listed with their signals and fold change. Expression levels of the representative SREBP-regulated genes (Hmgcr, Ldlr, and Fasn) are also shown.

to the inner leaflet $(14,16)$. Here, we demonstrated that the flipping of PtdCho by ATP11A causes lethal effects in humans and mice. Approximately 25\%-35\% of PtdCho in plasma membranes localizes to the inner leaflet $(34,35)$. However, electron microscopy findings with anti-PtdCho antibody revealed that the majority of PtdCho localizes to the outer leaflet, at least in red blood cells $(7,36)$. The harmful effects of the translocation of PtdCho to the inner leaflet of plasma membranes support its exclusive localization to the outer leaflet and suggest the presence of a phospholipid floppase or floppases that actively translocate PtdCho from the inner to the outer leaflet.

The Q84E mutation of hATP11A did not block the ability to translocate PtdSer and PtdEtn but allowed the molecule to translocate PtdCho. The amino acid Q84 localizes to the phospholipid "entry gate" in the first transmembrane segment (20) and appears to coordinate its substrate, PtdSer $(10,37)$. MD simulations indicated that the replacement of Q84 by Glu in ATP11C did not collapse its longitudinal cleft structure to capture PtdSer and PtdEtn; however, its negative charge stabilized binding to the choline moiety of PtdCho via electrostatic interactions. We observed PtdCho flipping not only for the Q84E mutant of hATP11A, but also for the corresponding Q79E mutation of hATP11C. However, the corresponding Q107E mutation of hATP8A2 did not induce PtdCho flipping. The amino acid sequences flanking the Glu residue are well conserved between hATP11A and hATP11C, but not between hATP11A and hATP8A2 (Supplemental Figure 6A). MD simulations indicated that the arrangement of amino acids around the phospholipid entry gate significantly differed between hATP11C and hATP8A1 and influenced substrate specificity. In this regard, it would be interesting to examine the structure of the hATP10A that translocates PtdCho, but not PtdSer or PtdEtn (38).
The present results indicate that PtdCho flipped by Q84EhATP11A traveled intracellularly. Cells carry specific lipid exchange systems. For example, PITP $\alpha$ carries PtdCho from the plasma membrane to the ER, in turn transferring PtdIns from the ER to the plasma membrane (39). PtdSer moves in the other direction. That is, ORP5 and ORP8 transfer PtdSer from the ER to plasma membranes, and PtdIns4P (PI4P) from plasma membranes to the ER. In addition, PtdSer can be trapped by the polybasic domains of plasma membrane-localized proteins (40). This may explain why PtdCho, but not PtdSer, at the inner leaflet of plasma membranes traveled intracellularly. The PtdCho that reached the ER directly or indirectly upregulated the Sgms1 gene. This result appears to be consistent with previous findings showing that PtdCho at the ER regulated the lipid metabolic pathway via nuclear receptors $(41,42)$. It will be interesting to study how PtdCho regulates Sgms1 gene expression.

Despite various attempts (microarray, single-cell RNA-Seq, and slide-Seq), we have so far failed to detect cells with increased Sgms1 gene expression in vivo (K. Segawa and S. Nagata, unpublished observation). This may be because the upregulation of Sgms1 gene expression occurred in a small population of cells, or because the cells with high SGMS1 levels had been killed, for example, by SMase. Using in vivo imaging analysis (MALDI-IMS), we detected several tissues that carried elevated SM levels (the brain, lung, and labyrinth; Figure 8). These results may be attributed to the high expression levels of ATP11A in the cells of these tissues, the weak "floppase" activity translocating PtdCho from the inner to the outer leaflet, or the lack of mechanisms to degrade the excess SM. Regardless of the mechanism, we detected cerebral atrophy in the patient and neuronal degeneration in Atp11 $a^{884 E / W T}$ mice, indicating that the loss of PtdCho or enrichment of SM at the outer leaflet of plasma membranes exerted deleterious effects in vivo. 
A
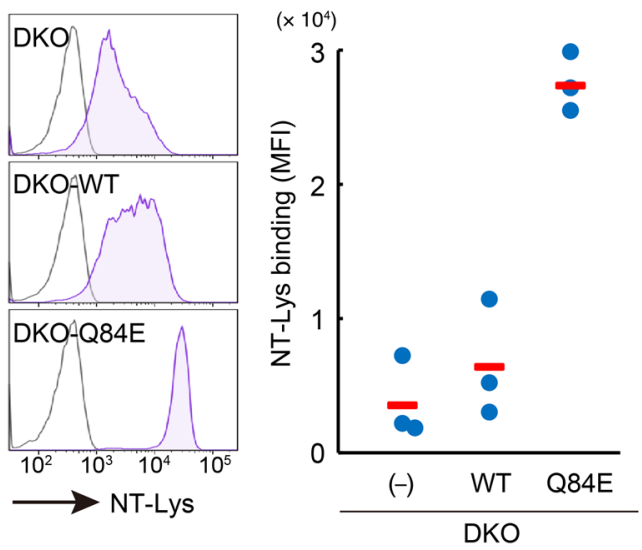

B

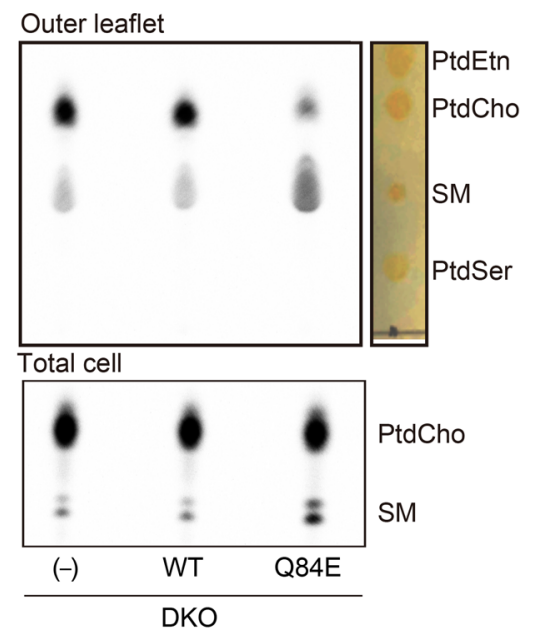

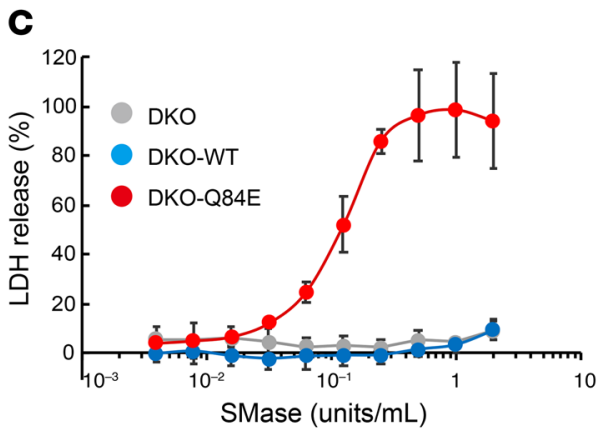
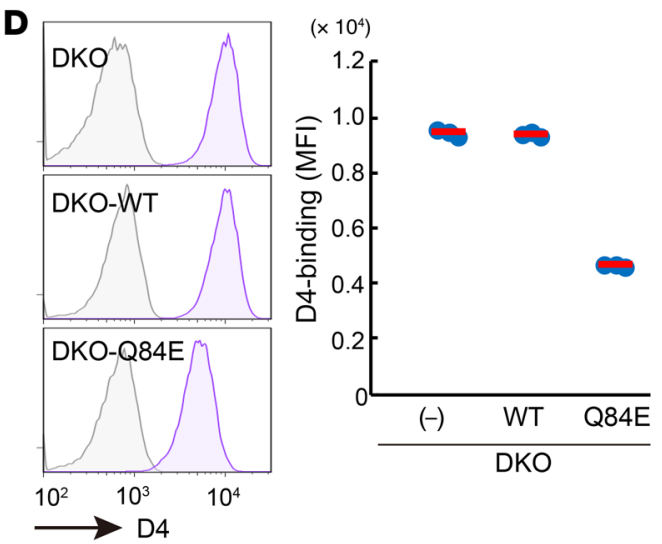

Figure 7. Replacement of PtdCho by $S M$ in the outer leaflet of plasma membranes. (A) DKO and its transformants expressing WT ATP11A or Q84E-ATP11A were stained with NT-Lys and analyzed by flow cytometry. The MFI is plotted on the right $(n=3)$. (B) The indicated cells were labeled with ${ }^{14} \mathrm{C}$-choline. Total cellular phospholipids (lower panel) and phospholipids extracted with MaCD (upper panel) were separated by TLC with standard lipids and visualized with an image analyzer or iodine vapor. (C) Cells were incubated with the indicated concentrations of nSMase for 5 minutes, and released LDH is expressed as the percentage released with $1 \%$ Triton-X $100(n=3)$. (D) Cells were stained with $\mathrm{mCher-}$ ry-D4, and the MFI is plotted $(n=3)$. (E) The total amount of the cholesteryl ester is plotted $(n=3)$. (F) Cells were cultured, and average cell numbers are plotted $(n=3)$. (G) The indicated cells were analyzed by flow cytometry, and their FSC/SSC profiles are shown. The average median FSC is shown with $\operatorname{SD}(n=4)$.

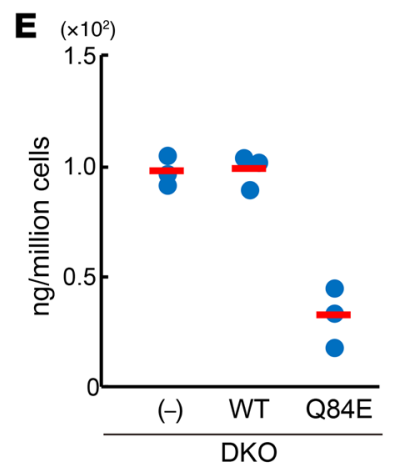

$\mathbf{F}$

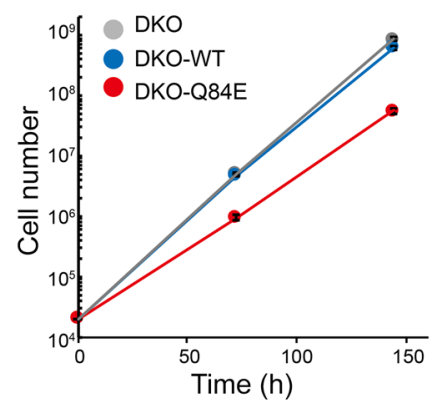

G

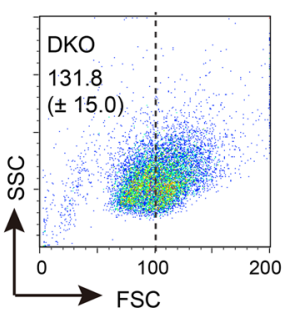

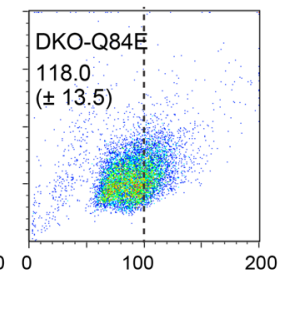

Affected cells may be susceptible to the cell lysis by SMase, since it is highly produced during inflammation (43-45). Or, the growth of these cells is retarded by abnormal cholesterol homeostasis (46). The Atp11a $a^{84 E / W T}$ neonate mice developed poorly and died within 20 weeks, but the mutant embryos developed until mid-gestation. In this regard, it may be worth noting that the oxygen stress generated during the fetal-to-neonate circulatory transition can activate SMase $(45,47)$. In conclusion, the present study showed that the appropriate distribution of PtdCho and SM in plasma membranes is essential for proper development in humans and mice.

\section{Methods}

Exome sequencing. Trio-based exome sequencing was performed for the patient and his parents using the HiSeq 2500 sequencer (Illumi- na) with 126 bp paired-end reads after enrichment with SureSelectXT Human All Exon V5 (50 Mb) Kits (Agilent Technologies). Reads were mapped against the hg19 human reference genome using Novoalign (version 2.08.05) (http://www.novocraft.com). Variants were called using Genome Analysis Toolkit software (version 1.6-13) (GATK). After quality filtering steps (Table 1), variants were annotated using ANNOVAR (48). Nonsynonymous SNVs, splice site variants, and indels were retained. To prioritize de novo variants, those in the following databases were excluded: 1000 Genomes Project (https:// www.internationalgenome.org/), NHLBI GO Exome Sequencing Project (https://evs.gs.washington.edu/EVS/), Genome Aggregation Database (gnomAD) (https://gnomad.broadinstitute.org/), Human Genetic Variation Database (http://www.hgvd.genome.med.kyoto-u. ac.jp/), and ToMMo 8.3KJPNv2 (https://jmorp.megabank.tohoku. 
A

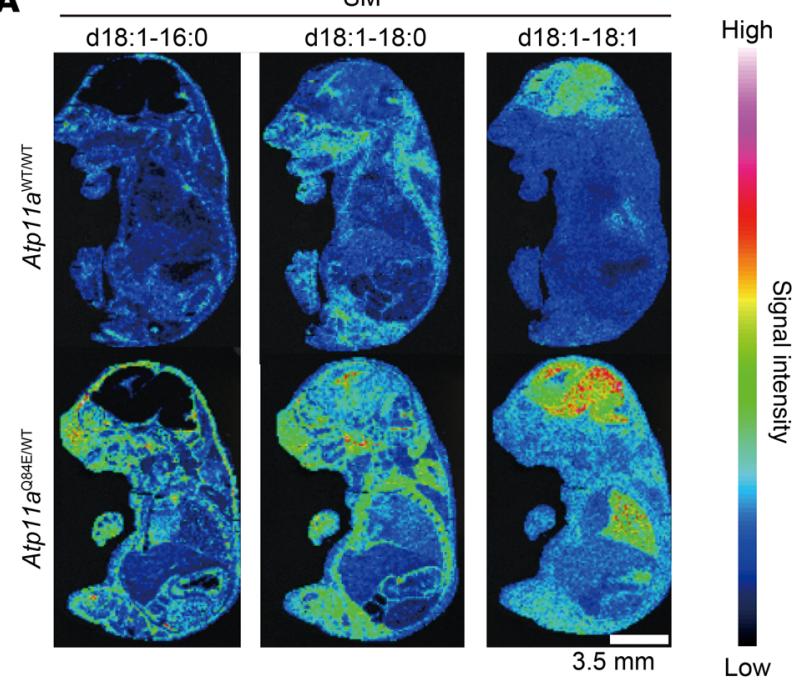

B

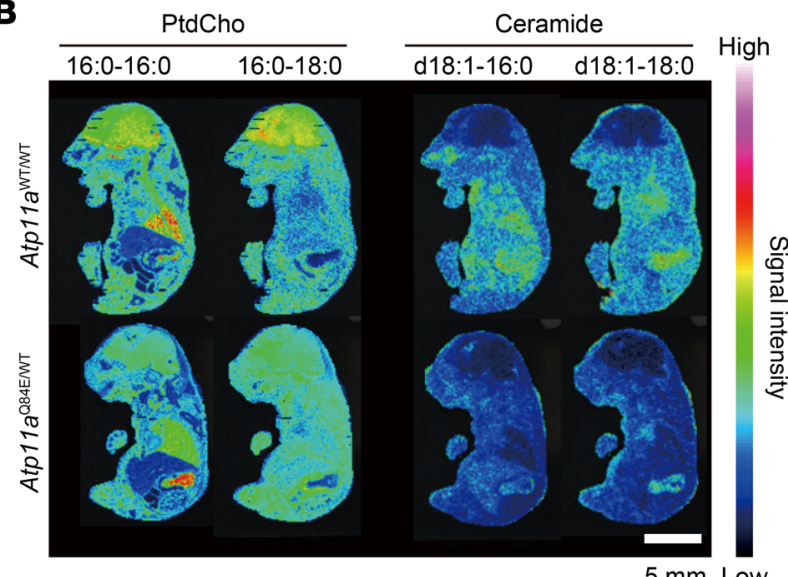

C
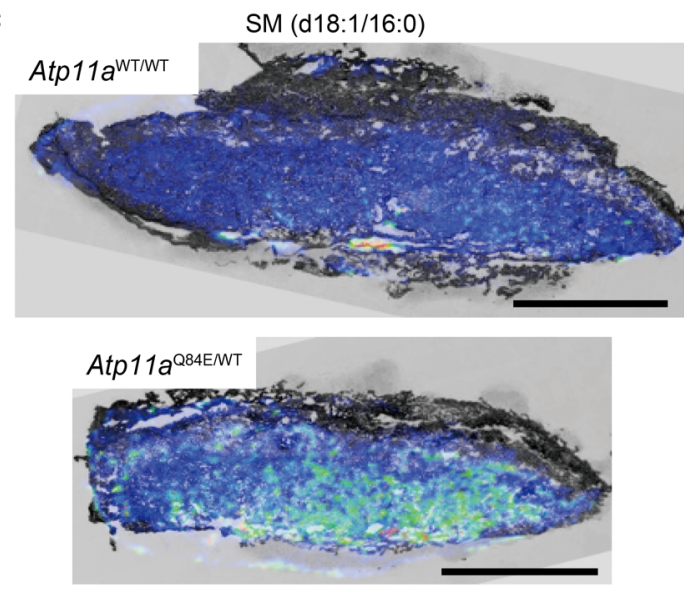

Figure 8. MALDI-IMS. Male Atp11afl/WT mice were mated with female CAG-Cre mice, and the E16 embryos (A and $\mathbf{B}$ ) and placentas (C) of the littermates were analyzed by MALDI-IMS. In C, images for SM (d18:1/16:0) were merged with bright-field images. Scale bars: $3.5 \mathrm{~mm}(\mathbf{A}), 5 \mathrm{~mm}(\mathbf{B})$, and $2 \mathrm{~mm}$ (C).

tion of the ATP11A gene (GenBank: NM_015205.3) thus identified in the patient was confirmed by Sanger sequencing.

Animal studies. C57BL/6J and ICR mice were obtained from Japan SLC or the Japan Central Laboratory for Experimental Animals. Atp11 $a^{f / W T}$ mice were generated by Unitech as a custom order. We established a conditional knockin allele (Atp11 $a^{f l}$ ) to express WT ATP11A in the absence of Cre recombinase and the Q84E mutant after Cre-mediated recombination. In brief, the mouse Atp11a gene (MGI: 1354735) was retrieved from BAC clones (RP23-81J20 and RP23-312I20) in RIKEN. A DNA carrying a part of intron 2 with a splice acceptor site, WT Atp11a cDNA encoding exons 3-29, triplet SV40 polyadenylation sequences, a neo gene flanked by flippase recognition target (FRT) sequences, and a loxP sequence were inserted into intron 2 of the Atp11a gene (Supplemental Figure 1). This was followed by insertion of exon 3 carrying the c.250C $>\mathrm{G}$ mutation, intron 3, exon 4, and a part of intron 4. A DNA for diphtheria toxin A (DT-A) was added at the $5^{\prime}$ terminus and introduced into embryonic stem (ES) cells (Bruce 4). ES cells carrying the targeted allele were selected by PCR and introduced into host embryos to generate chimeric mice. Crossing of chimeric mice with C57BL/6J mice produced Atp11 $a^{f l}$ mice. Atp11a $a^{Q 84 / W T}$ mice were produced by crossing male $A t p 11 a^{f l}$ mice with CAG-Cre mice (RIKEN: B6.Cg-Tg[CAGCre]CZ-MO2Osb). Mice were genotyped by PCR with the primers described in Supplemental Table 2.

IVF and embryo transfer. IVF was performed as described previously (49). In brief, sperm from Atp $11 a^{\mathrm{Q} 44 / W T}$ mice were incubated at $37^{\circ} \mathrm{C}$ for 2 hours in TYH medium (119.37 mM NaCl, $4.78 \mathrm{mM} \mathrm{KCl}, 1.19$ $\mathrm{mM} \mathrm{MgSO}_{4}, 1.19 \mathrm{mM} \mathrm{KH}_{2} \mathrm{PO}_{4}, 25.07 \mathrm{mM} \mathrm{NaHCO}, 1.71 \mathrm{mM} \mathrm{CaCl}$, $5.56 \mathrm{mM}$ D-glucose, $1 \mathrm{mM}$ sodium pyruvate, and $4 \mathrm{mg} / \mathrm{mL}$ AlbuMAX I [Thermo Fisher Scientific]). C57BL/6J female mice were injected i.p. with 5 units of pregnant mare serum gonadotropin 62 hours before IVF and 5 units of human chorionic gonadotropin 14 hours before IVF. Eggs were treated with $1 \mathrm{mg} / \mathrm{mL}$ hyaluronidase (MilliporeSigma) at $37^{\circ} \mathrm{C}$ for 5 minutes. Sperm were allowed to fertilize eggs at $37^{\circ} \mathrm{C}$ for 6 hours and were then incubated in TYH for 18 hours. Two-cell stage embryos were transferred to oviducts of pseudo-pregnant ICR females (0.5 days postcoitum [dpc]). For ICSI (50), sperm heads were separated from tails by applying a few piezo pulses and injected into oocytes using a piezo micro manipulator (PrimeTech). Embryos were transferred into the oviducts as described above.

Plasmids. pMXs-puro and pGag-pol-IRES-bsr (51) were obtained from T. Kitamura (University of Tokyo). pCMV-VSV-G, pQE30/His6mRFP-NT-Lys, and pET28/His6-mCherry-D4 were obtained from RIKEN.pAdVAntage and pX330 were purchased from Thermo Fisher Scientific and Addgene, respectively. cDNAs for hCDC50A (NM_018247.3), hATP11A (NM_015205.2), hATP11C (XM_005262405.1), and hATP8A2 (NM_016529.4) were prepared by PCR (14), tagged with FLAG or GFP at the $\mathrm{C}$-terminus, and introduced into pMXs-puro. hATP11A cDNA carrying the c. $250 C>G$ mutation was prepared by PCR from the patient's RNA with the primers $5^{\prime}$-GGCTTAATTAAGGAGGAGCCATGGACT- 
GCAGCCTCG-3' and 5'-TTCATCGATTTTTCCACGGC-3' (the restriction enzyme recognition sites are underlined). The PCR product was digested with PacI and ClaI and used to replace WT hATP11A cDNA in pMXs-puro-hATP11A-GFP. Other cDNAs carrying the point mutation of $\mathrm{hATP11A}, \mathrm{h} A T P 8 A 2$, and $\mathrm{h} A T P 11 \mathrm{C}$ were prepared by recombinant PCR (52) using the primers described in Supplemental Table 2 and sequenced by Sanger sequencing.

Cell lines. HEK293T cells (American Type Culture Collection [ATCC]: CRL-3216) were cultured in DMEM containing 10\% FCS (Gibco, Thermo Fisher Scientific). W3 is a WR19L cell (ATCC: TIB-52) transformant expressing mouse Fas (53). The W3 cell line and its derivative (DKO) lacking the Atp11a and Atp11c genes (17) were cultured in RPMI 1640 with $10 \%$ FCS. Mouse Ba/F3 cells (54) were cultured in RPMI 1640 with $10 \%$ FCS containing $45 \mathrm{U} / \mathrm{mL}$ mouse IL-3. Cells were stably transformed as described previously (14). In brief, pMXs-puro carrying the GFP-tagged cDNA was introduced into HEK293T cells by Fugene 6 (Promega) with pGag-pol-IRES-bsr, pCMV-VSV-G, and pAdVAntage. Two days later, retroviruses in the supernatant were collected by centrifugation and used for infection. Cells were cultured in the presence of $1 \mu \mathrm{g} / \mathrm{mL}$ puromycin, and GFP-positive cells were sorted with FACSAria II (BD Biosciences). Human skin fibroblasts (ASF-43L) from healthy individuals were obtained from RIKEN. The patient's skin fibroblasts were prepared by mincing the skin biopsy samples and culturing them in DMEM with $10 \%$ FCS.

Antibodies and reagents. The rabbit HRP anti-GFP Ab (catalog 5987) was from MBL International. Anti-FLAG M2-conjugated magnetic beads (catalog M8823) were purchased from MilliporeSigma. Mouse anti-SGMS1 mAb (clone E-5: sc-166436) was from Santa Cruz Biotechnology. HRP-goat anti-mouse IgG2a heavy chain (catalog ab97245) was obtained from Abcam. Neutral SMase from B. cereus was from MilliporeSigma. DRAQ5, ER-Tracker Blue-White DPX, Hoechst 33342, and SYTOX Blue were from Thermo Fisher Scientific. NBD-PS, NBDPE, NBD-PC, NBD-SM, NBD-GalCer, 12:0 NBD-LysoPC, POPS, POPE, POPC, 18:0 SM (d18:1/18:0), TopFluor PC, TopFluor PS, and porcine brain sphingomyelin were obtained from Avanti Polar Lipids, and 2,6-Di-O-methyl- $\alpha$-cyclodextrin was from purchased from Wako.

Preparation of NT-Lys and perfringolysin domain 4. To produce red fluorescent protein-tagged (RFP-tagged) NT-Lys and mCherrydomain 4 (D4) of perfringolysin (mCherry-D4), the DNA fragment coding for His6-NT-Lys-RFP or His6-mCherry-D4 (28, 55) was inserted into the pCold I vector (Takara) and introduced into chaperone-competent E. coli (pGro7/BL21; Takara). Transformants were grown until the $\mathrm{OD}_{600}$ reached 0.5. After the addition of $\mathrm{L}$-arabinose and isopropyl $\beta$-D-thiogalactopyranoside (IPTG), cells were cultured at $15^{\circ} \mathrm{C}$ for 24 hours. The cells were disrupted by sonication in $100 \mathrm{~mL}$ buffer A (50 mM Tris-HCl buffer [pH 8.0], $150 \mathrm{mM} \mathrm{NaCl}$, and $0.1 \mathrm{mM}$ DTT) containing an EDTA-free protease inhibitor cocktail (Nacalai). After removal of cell debris, the supernatant was loaded onto Ni-NTA Superflow (QIAGEN) equilibrated with buffer A. The column was washed with buffer B (50 mM Tris-HCl buffer [pH 8.0], $500 \mathrm{mM}$ $\mathrm{NaCl}$ ), and bound proteins were eluted with elution buffer $(50 \mathrm{mM}$ Tris-HCl buffer [pH 7.4], $150 \mathrm{mM} \mathrm{NaCl}$ ) containing 10, 100, 300, or $500 \mathrm{mM}$ imidazole in a stepwise manner. The fractions containing NT-Lys or mCherry-D4 were concentrated using Amicon Ultra 10K (MilliporeSigma) and dialyzed against PBS.

Flippase assay. Flippase activity was assayed as described previously $(13,14)$. In brief, $2 \times 10^{6}$ cells were incubated at $20^{\circ} \mathrm{C}$ with $0.25-$
1.0 $\mu \mathrm{M}$ NBD phospholipids in $600 \mu \mathrm{L}$ HBSS containing $1 \mathrm{mM} \mathrm{MgCl}_{2}$ and $2 \mathrm{mM} \mathrm{CaCl}_{2}\left(\mathrm{HBSS}^{++}\right)$. Cells were collected by centrifugation, suspended in HBSS containing $5 \mathrm{mg} / \mathrm{mL}$ fatty acid-free BSA to remove nonincorporated NBD lipids, and analyzed using a FACSCanto II (BD Biosciences). Dead cells were omitted from the analysis by standard forward scatter/side scatter (FSC/SSC) gating. The fluorescence that was not removed from cells by the BSA treatment reflected the flipping activity and was shown as NBD phospholipid incorporation.

The flipping activity with TopFluor-conjugated lipids was assayed using the M $\alpha \mathrm{CD}$-mediated lipid exchange system (25). To prepare SM-loaded M $\alpha \mathrm{CD}, 5.7 \mathrm{mg}$ SM was dried under a nitrogen stream for 1 hour, suspended in $4.5 \mathrm{~mL} \mathrm{RPMI}$ medium prewarmed to $70^{\circ} \mathrm{C}$, and incubated at $70^{\circ} \mathrm{C}$ for 5 minutes. After adding $500 \mu \mathrm{L}$ of $40 \mathrm{mM} \mathrm{M \alpha CD}$ in PBS, the solution was incubated at $37^{\circ} \mathrm{C}$ for 1 hour, centrifuged at $10,000 \mathrm{~g}$ for 5 minutes, and the supernatant was used as SM-loaded $\mathrm{M} \alpha \mathrm{CD}$. Cells $\left(2 \times 10^{6}\right.$ cells $)$ were incubated on ice for 10 minutes in $1 \mathrm{~mL}$ HBSS containing $5 \mu \mathrm{M}$ TopFluor-PC, resuspended in $\mathrm{HBSS}^{++}$that had been prewarmed to $20^{\circ} \mathrm{C}$, and incubated at $20^{\circ} \mathrm{C}$ for 20 minutes. Cells were collected by centrifugation, suspended in $500 \mu \mathrm{L}$ SM-loaded $\mathrm{M} \alpha \mathrm{CD}$, incubated at $20^{\circ} \mathrm{C}$ for 1 hour, and analyzed by flow cytometry using FACSCanto II or observed under a confocal microscope (FV1000D; Olympus).

Quantification of cholesterol, staining for SM and cholesterol, and treatment with SMase. Cellular cholesterol and the cholesteryl ester were quantified using the Total Cholesterol and Cholesteryl Ester Colorimetric/Fluorometric Assay kit (BioVision). For staining with NT-Lys or His6-mCherry-D4, $1 \times 10^{5}$ to $2 \times 10^{5}$ cells were incubated on ice for 30 minutes with $10 \mu \mathrm{g} / \mathrm{mL}$ His6-NT-Lys-mRFP or His6-mCherry-D4 in HBSS containing $250 \mathrm{nM}$ SYTOX Blue and analyzed using the FACSCanto II. For treatment with SMase, $3 \times 10^{4}$ cells were incubated at $37^{\circ} \mathrm{C}$ for 5 minutes with neutral SMase or $1 \%$ Triton-X100 in $100 \mu \mathrm{L}$ DMEM on 96-well round-bottomed dishes. LDH activity was measured using the LDH Cytotoxicity Detection Kit (Takara) with $60 \mu \mathrm{L}$ aliquots of the supernatant.

Labeling cells with ${ }^{14} \mathrm{C}$-choline, lipid extraction, and TLC. Cells were cultured for 40 hours in $12 \mathrm{~mL}$ RPMI 1640 containing 10\% FCS and $1 \mu \mathrm{Ci}$ choline chloride [methyl $-^{-14} \mathrm{C}$ ] $(55 \mathrm{mCi} / \mathrm{mmol}$, American Radiolabeled Chemicals [ARC]). For lipids in the outer leaflet of plasma membranes, $3 \times 10^{6}$ to $5 \times 10^{6}$ cells were suspended in $1 \mathrm{~mL}$ SM-loaded $\mathrm{M} \alpha \mathrm{CD}$ solution and incubated at $37^{\circ} \mathrm{C}$ for 1 hour. After removal of $\mathrm{M} \alpha \mathrm{CD}$ by centrifugation, supernatants were vigorously mixed with a 1 $\mathrm{mL}$ hexane/isopropanol mixture (3:2, vol/vol), and the organic phase was collected. Lipids in the water phase were re-extracted with the same treatment, combined with the first extract, dried under a nitrogen stream, and dissolved in $25 \mu \mathrm{L}$ of a 1:1 (v/v) mixture of methanol/ chloroform. Extracted lipids (5000 disintegrations per minute [dpm]) were separated on an HP-TLC Silica Gel 60 Plate (Merck) with chloroform/methanol/ammonium hydroxide $(65: 25: 5$, vol/vol/vol) as a solvent and visualized with an imaging plate detector (FLA-7000, Fujifilm). Lipids were also visualized by exposure to iodine vapor, collected with a spatula, and mixed with scintillator cocktail (Clear-sol I; Nacalai Tesque). Radioactivity was measured using the Tri-Carb 4810 scintillation counter (PerkinElmer).

LC-MS/MS and MALDI-IMS. For LC-MS/MS analysis, lipids were extracted from the cells by methanol and analyzed by LC-electrospray ionization-MS/MS using a TSQ-Vantage (Thermo Fisher Scientific) at Japan Lipid Technologies as a custom order. For MALDI-IMS, samples 
were embedded in Super Cryoembedding Medium (Section-Lab) and frozen in powdered dry ice. Sections ( $8 \mu \mathrm{m}$ thick) were prepared using a Leica CM3050 cryostat, attached to indium tin oxide-coated glass slides (Bruker Daltonics), and coated with 2,5-dihydroxybenzoic acid $(50 \mathrm{mg} / \mathrm{mL}$ in $80 \%$ ethanol) by spraying with an artistic brush (Procon Boy FWA Platinum, Mr. Hobby). The ultraflextreme MALDI-TOF mass spectrometer (Bruker Daltonics) was equipped with an Nd:YAG laser. Data were acquired in the positive reflectron mode with raster scanning by a pitch distance of $50 \mu \mathrm{m}$. Signals with an $\mathrm{m} / \mathrm{z}$ between 400 and 900 were collected in the continuous accumulation of selected ions mode and expressed as the image using FlexImaging 4.1 software (Bruker Daltonics).

Microscopic analysis and TUNEL staining. Mouse embryos were fixed at $4^{\circ} \mathrm{C}$ in $0.1 \mathrm{M}$ phosphate buffer ( $\mathrm{pH}$ 7.4) containing $2 \%$ formaldehyde and $2.5 \%$ glutaraldehyde, postfixed for 1 hour with $1 \% \mathrm{OsO}_{4}$, dehydrated, and embedded in TAAB Epon 812 (Nissin-EM). Sections ( $2 \mu \mathrm{m}$ thick) were stained with toluidine blue and observed under a BX63 Olympus microscope. For electron microscopy studies, fixed brains were cut into small pieces, dehydrated, and embedded in Epon 812. Silver sections (80 nm thick) were prepared with an ultramicrotome (Ultracut UCT, Leica), stained with uranyl acetate and lead citrate, and observed with an electron microscope (HT7700, Hitachi Ltd.). For TUNEL staining, mouse brains were fixed with $4 \%$ paraformaldehyde, embedded in paraffin, and sliced into $5 \mu \mathrm{m}$ thick sections. Sections were subjected to TUNEL staining using the In situ Cell Death Detection Kit, TMR red (Roche), followed by observation with a fluorescence microscope (BIOREVO BZ-9000, Keyence). Six to 7 images that covered the whole-brain tissues were obtained, and the TUNEL-positive foci per brain tissue were counted using Hybrid Cell Count on BZ-H4C (Keyence) image analysis software.

Mouse MRI analysis. Mouse MRI was performed using the Avance III BioSpec 117/11 system (Bruker) equipped with a ${ }^{1} \mathrm{H}$ QD coil. During the analysis, mice were anesthetized and their body temperature maintained at $37^{\circ} \mathrm{C}$ with a warm-water circulating system. The fast spin-echo protocol was applied for $\mathrm{T} 2$-weighted imaging. Images were converted to the DICOM format and visualized using Image (NIH). The MRI parameters were as follows: time of repetition/time of echo (TR/TE) of 3000/48 ms; mean number of 4; image matrix of $200 \times$ 200 (processed to $256 \times 256$ by 0-filling); and field of view of $26 \times 26$ $\mathrm{mm}$, with 14 slices of $0.5 \mathrm{~mm}$ thickness without a gap between slices. Two sets of images acquired under the above conditions with a 0.25 $\mathrm{mm}$ offset gap along the axis were merged and processed as 1 imaging data set of 28 slices. Brain volumes were calculated using ImageJ by multiplying the summation area from the olfactory bulb to the cerebellum and thickness.

Purification of flippase and ATPase assay. The flippase was purified, and its ATPase activity was measured as described before $(13,14)$. For WT hATP11A, HEK293T cells were transfected with pEF-BOS-hATP11A-FLAG and pEF-BOS-hCDC50A-GFP and cultured for 2 days, whereas, for Q84E-hATP11A, the Ba/F3 transformants expressing hATP11A-Q84E-FLAG and hCDC50A-GFP were established. 293T and $\mathrm{Ba} / \mathrm{F} 3$ cells were lysed with solubilization buffer (40 mM MES/ Tris buffer [pH 7.0], $5 \mathrm{mM} \mathrm{MgCl}, 150 \mathrm{mM} \mathrm{NaCl}, 10 \%$ glycerol, 0.5 mM DTT, 0.5\% lauryl maltose-neopentyl glycol [LMNG], and a cocktail of protease inhibitors). After removal of cell debris, cell lysates were mixed with anti-FLAG M2- beads, and bound proteins were eluted with $160 \mu \mathrm{g} / \mathrm{mL} 3 \times$ FLAG Peptide (MilliporeSigma) in $40 \mathrm{mM}$
MES/Tris buffer (pH 7.0) consisting of $150 \mathrm{mM} \mathrm{NaCl}, 10 \%$ glycerol, 0.5 mM DTT, and 0.05\% LMNG.

Phospholipids were dissolved in $0.5 \%$ lauryl maltose neopentyl glycol (LMNG) by incubation at $50^{\circ} \mathrm{C}$ for 30 minutes. The purified flippase (10 ng) was successively incubated at room temperature for 10 minutes and at $37^{\circ} \mathrm{C}$ for 20 minutes in $40 \mathrm{mM} \mathrm{MES/Tris} \mathrm{buffer}(\mathrm{pH}$ 7.0) containing $5 \mathrm{mM} \mathrm{MgCl}, 150 \mathrm{mM} \mathrm{NaCl}, 600 \mu \mathrm{M}$ ATP, $10 \%$ glycerol, $5 \mathrm{mM}$ DTT, $30 \mu \mathrm{M}$ phospholipids, and 0.05\% LMNG. Samples were mixed with malachite green molybdate, and the resulting malachite green-ammonium molybdate phosphate complex was detected at $610 \mathrm{~nm}$ using a Micro Plate Reader (Infinite M200; TECAN).

Western blotting, microarray analysis, and real-time RT-PCR. Cells were lysed with $50 \mathrm{mM}$ HEPES-NaOH buffer ( $\mathrm{pH}$ 8.0) containing 1\% $\mathrm{NP}-40,0.1 \%$ SDS, $0.5 \%$ sodium deoxycholate, $150 \mathrm{mM} \mathrm{NaCl}$, and a protease inhibitor cocktail. After removal of cell debris by centrifugation, a one-fourth volume of $5 \times$ SDS sample buffer $(330 \mathrm{mM}$ Tris$\mathrm{HCl}$ buffer [pH 6.8], 10\% SDS, 50\% glycerol, 5\% $\beta$-mercaptoethanol, and $0.015 \%$ bromophenol blue) was added and incubated at $25^{\circ} \mathrm{C}$ for 20 minutes. Proteins were separated by $7.5 \%$ or $10 \%-20 \%$ PAGE (Nacalai) together with Precision Plus Protein Standards (Bio-Rad) and then transferred onto PVDF membranes (MilliporeSigma). Membranes were probed with HRP-conjugated rabbit anti-GFP or mouse anti-SGMS1 followed by HRP-conjugated goat anti-mouse IgG2a. Peroxidase activity was detected using the Western Lightning ECL system (PerkinElmer).

Microarray analysis was performed using the GeneChip 3' IVT PLUS Reagent Kit (Thermo Fisher Scientific) as a custom order at Takara Bio. In real-time RT-PCR, RNA was reverse-transcribed with a High Capacity RNA-to-cDNA kit (Thermo Fisher Scientific), and cDNA was amplified in a reaction mixture of LightCycler 480 SYBR Green I Master (Roche Diagnostics). Data are available from the NCBI's Gene Expression Omnibus database (GEO GSE181171). Primers for real-time RT-PCR are described in Supplemental Table 2.

Data-dependent LC-MS/MS analysis of immunoprecipitated GFPtagged hATP11A. DKO cells stably expressing GFP-tagged WT or Q84E-hATP11A were lysed in RIPA buffer (20 mM HEPES-NaOH [pH7.5], $150 \mathrm{mM} \mathrm{NaCl}, 1 \mathrm{mM}$ EGTA, $1 \mathrm{mM} \mathrm{MgCl}_{2}, 0.25 \%$ sodium deoxycholate, $0.2 \%$ SDS, and $1 \%$ NP-40) supplemented with a protease inhibitor cocktail and a PhosSTOP phosphatase inhibitor cocktail (Roche). After centrifugation at 20,000 $g$ at $4^{\circ} \mathrm{C}$ for 15 minutes, supernatants were incubated with a $4 \mu \mathrm{L}$ slurry of GFP-Trap_MA (ChromoTek) at $4^{\circ} \mathrm{C}$ for 2 hours. The beads were washed 4 times with RIPA buffer and twice with $50 \mathrm{mM}$ ammonium bicarbonate. Proteins on the beads were digested with $200 \mathrm{ng}$ chymotrypsin (Roche) at $25^{\circ} \mathrm{C}$ for 16 hours. The digests were reduced, alkylated, acidified, and desalted using GL-Tip SDB (GL Sciences). The eluates were evaporated in a SpeedVac concentrator and dissolved in $0.1 \%$ trifluoroacetic acid and $3 \%$ acetonitrile (ACN). LC-MS/MS analysis was performed on an EASY-nLC 1200 UHPLC connected to an Orbitrap Fusion mass spectrometer through a nanoelectrospray ion source (Thermo Fisher Scientific). Peptides were separated on a C18 reversed-phase column $(75 \mu \mathrm{m} \times 150 \mathrm{~mm})$ (Nikkyo Technos) with a linear 4\%-36\% ACN gradient for 0-100 minutes, followed by an increase to $80 \%$ ACN for 10 minutes. The mass spectrometer was operated in the data-dependent acquisition mode with a maximum duty cycle of 3 seconds. MS1 spectra were measured with a resolution of 60,000, an automatic gain control (AGC) target of $4 \times 10^{5}$, and a mass range from 350 to $1500 \mathrm{~m} / z$. Collision-induced dissociation 
(CID) MS/MS spectra were acquired in the Orbitrap with a resolution of 30,000, an AGC target of $5 \times 10^{4}$, an isolation window of $1.6 \mathrm{~m} / \mathrm{z}$, a maximum injection time of $100 \mathrm{~ms}$, and a normalized collision energy of 35. Dynamic exclusion was set to 5 seconds. Raw data were analyzed against the SwissProt database restricted to Mus musculus supplemented with human ATP11A (WT and Q84E) and EGFP protein sequences using Proteome Discoverer version 2.4 (Thermo Fisher Scientific) with the SEQUEST HT search engine. The search parameters were as follows: (a) chymotrypsin as an enzyme with up to 3 missed cleavages; (b) precursor mass tolerance of $10 \mathrm{ppm}$; (c) fragment mass tolerance of $0.02 \mathrm{Da}$; (d) the carbamidomethylation of cysteine as a fixed modification; and (e) acetylation of the protein N-terminus and oxidation of methionine as variable modifications. Peptides were filtered at an FDR of $1 \%$ using the percolator node.

Parallel reaction monitoring analysis of ATP11A peptides from mouse placenta. The frozen placenta was disrupted using an ultrasonic homogenizer (Q55, QSonica) in $6.5 \mathrm{~mL}$ of $10 \mathrm{mM}$ Tris-HCl buffer $\mathrm{pH}$ 7.5) supplemented with $0.5 \mathrm{mM}$ DTT and a protease inhibitor cocktail (buffer $\mathrm{C}$ ). After addition of an equal volume of buffer $\mathrm{C}$ containing 0.5 M sucrose, $0.1 \mathrm{M} \mathrm{KCl}, 10 \mathrm{mM} \mathrm{MgCl}_{2}$, and $2 \mathrm{mM} \mathrm{CaCl}_{2}$, the mixture was incubated on ice for 15 minutes with 10 units/mL benzonase (Merck) and serially centrifuged at $800 \mathrm{~g}$ and $8000 \mathrm{~g}$ each at $4^{\circ} \mathrm{C}$ for 10 minutes. The supernatant was then centrifugated at $100,000 \mathrm{~g}$ for 1 hour, and the precipitates were recovered as a crude membrane fraction.

The placenta membrane fractions were lysed in $1 \times$ SDS sample buffer, incubated at $25^{\circ} \mathrm{C}$ for 20 minutes, separated by $5 \%-20 \%$ SDSPAGE, and stained with Coomassie brilliant blue (CBB). Bands with a MW between 100 and $130 \mathrm{kDa}$ were cut out and digested in gel with chymotrypsin at $25^{\circ} \mathrm{C}$ for 16 hours as described previously (56). Several selected peptides of ATP11A (WT and Q84E) were measured by parallel reaction monitoring (PRM) (19). Targeted CID MS/MS scans were acquired by a time-scheduled inclusion list at a resolution of 60,000 , an AGC target of $1.5 \times 10^{5}$, an isolation window of $1.6 \mathrm{~m} / \mathrm{z}$, a maximum injection time of 1 second, and a normalized collision energy of 35 . The time alignment of the transitions was performed using Skyline software.

Molecular model and MD simulations. We used the cryo-EM structures of hATP11C (PDB: 7BSU; ref. 23) and hATP8A1 (PDB: 6K7M; ref. $10)$ in the PtdSer-occluded E2- $\mathrm{P}_{\mathrm{i}}$ state. Hydrogen atoms were generated and energetically optimized with the CHARMM program (version $40 b)$ (57). The positions of all nonhydrogen atoms were fixed, and all titratable groups were maintained in their standard protonation state at $\mathrm{pH}$ 7. The hATP11C-CDC50A or hATP8A1-CDC50A flippase complex in the PtdSer-occluded E2- $\mathrm{P}_{\mathrm{i}}$ state (WT PtdSer) was embedded in a lipid bilayer consisting of approximately 1000 POPC molecules and soaked in approximately 180,000 water molecules. Fifteen chloride ions were added to the model using the CHARMM-GUI program (58). After structural optimization with positional restraints on heavy atoms of the WT PtdSer assembly, (a) the system was heated from 0.1 to $300 \mathrm{~K}$ over $5.5 \mathrm{ps}$ with intervals of $0.01 \mathrm{fs}$, equilibrated at $300 \mathrm{~K}$ for $1 \mathrm{~ns}$ with $0.5 \mathrm{fs}$ intervals, and annealed from 300 to $0 \mathrm{~K}$ over 5.5 ps with intervals of $0.01 \mathrm{fs}$. (b)The same procedure was repeated with positional restraints on the heavy atom of the protein backbone. (c) The same procedure, except for the annealing process, was repeated without positional restraints on any atoms. (d) The WT PtdSer structure was obtained after the system was equilibrated at $300 \mathrm{~K}$ for 67.5 ns with intervals of 1.5 fs. For WT PtdCho and WT PtdEtn, initial WT lipid structures were modeled on the basis of the WT PtdSer struc- ture, and steps (c) and (d) were repeated. Mutant lipid structures were modeled on the basis of the WT lipid structures, and steps (c) and (d) were repeated. All equilibrating simulations were conducted using the NAMD program (version 2.12; ref. 59). The SHAKE algorithm (60) was used for hydrogen constraints with a time step of $1.5 \mathrm{fs}$ where applicable. The Langevin thermostat and piston were used for temperature and pressure control, respectively (61). The binding energy between the lipid and flippase complex (excluding cavity-filling water molecules) was calculated on the basis of 200 conformations in the last 22.5 ns MD simulation using the Molecular Operating Environment (MOE) program (Chemical Computing Group). The population of the H-bond pattern at the binding moiety was analyzed from 3000 conformations in the last $22.5 \mathrm{~ns}$ MD simulation (refer to Supplemental Table 1 for the number of water molecules that formed $\mathrm{H}$-bonds with lipids).

Statistics. The significance of differences was calculated with a 2-tailed Student's $t$ test. Differences were considered significant when the $P$ value was less than 0.05 .

Study approval. The use of patient samples was approved by the ethics committees of the Research Institute for Microbial Diseases at Osaka University (protocol no. 30-2) and the Tohoku University Graduate School of Medicine (protocol no. 2015-1-694). Written informed consent was obtained from the parents of the patient. Mice were maintained under specific pathogen-free conditions at Osaka University. Experiments were conducted according to protocols approved by the IACUC of the Research Institute of Microbial Diseases at Osaka University.

\section{Author contributions}

$\mathrm{KS}, \mathrm{AK}, \mathrm{SK}$, and SN conceived and designed the project. AK, SI, and SK identified the ATP11A mutation in the patient. KS, AK, KY, YO, T Nishimura, MM, SI, and WS performed the experiments. YS and MS performed MALDI-IMS analysis. T Noji, K Hiraga, and HI conducted MD simulations. CS and YU performed the electron microscopic analyses. K Haginoya and YK collected and analyzed clinical data and provided patient samples. FS performed the MRI analysis. HK and KN performed LC-MS/MS-based targeted proteomics analysis. MI conducted IVF and ICSI experiments. KS, $\mathrm{AK}, \mathrm{SK}, \mathrm{YS}, \mathrm{HN}, \mathrm{HI}$, and SN wrote the manuscript. All authors read and approved the manuscript.

\section{Acknowledgments}

This work was supported by Grants-in-Aid from the Japan Society for the Promotion of Science (18H02615, 16H01360, and $17 \mathrm{H05506}$, to KS; $15 \mathrm{H} 05785$ and $21 \mathrm{HO} 4770$, to SN; and JP18H05155, JP18H01937, JP2OH03217, and JP2OH05090, to HI); Grants-in-Aid from Core Research for Evolutional Science and Technology of the Japan Science and Technology Agency (JPMJCR14M4, to SN; JPMJCR1656, to HI); and Grants-in-Aid from the Initiative on Rare and Undiagnosed Diseases of the Japan Agency for Medical Research and Development (JP17ek0109151, to SK; JP18ek0109288, to WS). This work was also supported by Joint Usage and Joint Research Programs of the Institute of Advanced Medical Sciences, Tokushima University (to SN), and the Program for the Creation of Interdisciplinary Research at the Frontier Research Institute for Interdisciplinary Sciences of Tohoku University (to AK and WS). We are grateful to the patient and his family. We thank K. Hasegawa, Y. Chiba, and K. Ito for their technical assistance, and M. Fujii and M. Kamada for their secretarial 
assistance. We thank M. Shirota, R. Funayama, and K. Nakayama (United Centers for Advanced Research and Translational Medicine, Tohoku University Graduate School of Medicine) and T. Niihori and Y. Aoki (Department of Medical Genetics, Tohoku University Graduate School of Medicine) for the exome-sequencing analysis. We thank the staff at the Biomedical Research Core of the Tohoku University Graduate School of Medicine and the Biomedical Research Unit of Tohoku University Hospital.
Address correspondence to: Shigekazu Nagata, Laboratory of Biochemistry \& Immunology, Immunology Frontier Research Center, Osaka University, 3-1 Yamada-oka, Suita, Osaka 565-0871, Japan; Phone: 81.6.6879.4953; E-mail: snagata@ifrec.osaka-u.ac.jp. KS’s present address is: Department of Medical Chemistry, Medical Research Institute, Tokyo Medical and Dental University, Tokyo, Japan. TN's present address is: Regenerative Medicine Division, Cell Processing Department, Tella Inc., Kyoto, Japan.
1. Harayama T, Riezman H. Understanding the diversity of membrane lipid composition. Nat Rev Mol Cell Biol. 2018;19(5):281-296

2. Vance JE. Phospholipid synthesis and transport in mammalian cells. Traffic. 2015;16(1):1-18.

3. Yang Y, et al. Phospholipid subcellular localization and dynamics. J Biol Chem. 2018;293(17):6230-6240.

4. Das A, et al. Three pools of plasma membrane cholesterol and their relation to cholesterol homeostasis. Elife. 2014;3:e02882.

5. Mitra K, et al. Modulation of the bilayer thickness of exocytic pathway membranes by membrane proteins rather than cholesterol. Proc Natl Acad Sci US A. 2004;101(12):4083-4088.

6. van Meer G, et al. Membrane lipids: where they are and how they behave. Nat Rev Mol Cell Biol. 2008;9(2):112-124

7. Murate M, et al. Transbilayer distribution of lipids at nano scale. JCell Sci. 2015;128(8):1627-1638.

8. Coleman JA, et al. Mammalian P4-ATPases and $\mathrm{ABC}$ transporters and their role in phospholipid transport. Biochim Biophys Acta. 2013;1831(3):555-574.

9. Andersen JP, et al. P4-ATPases as phospholipid flippases-structure, function, and enigmas. Front Physiol. 2016;7:275.

10. Hiraizumi M, et al. Cryo-EM structures capture the transport cycle of the P4-ATPase flippase. Science. 2019;365(6458):1149-1155.

11. Nakanishi H, et al. Crystal structure of a human plasma membrane phospholipid flippase. J Biol Chem. 2020;295(30):10180-10194.

12. Nagata $\mathrm{S}$, et al. Exposure of phosphatidylserine on the cell surface. Cell Death Differ. 2016;23(6):952-961.

13. Segawa K, et al. The CDC50A extracellular domain is required for forming a functional complex with and chaperoning phospholipid flippases to the plasma membrane. J Biol Chem. 2018;293(6):2172-2182.

14. Segawa K, et al. Human type IV P-type ATPases that work as plasma membrane phospholipid flippases and their regulation by caspase and calcium. J Biol Chem. 2016;291(2):762-772.

15. Tanaka K, et al. Functions of phospholipid flippases. J Biochem. 2011;149(2):131-143.

16. Segawa K, et al. Caspase-mediated cleavage of phospholipid flippase for apoptotic phosphatidylserine exposure. Science. 2014;344(6188):1164-1168.

17. Segawa K, et al. Phospholipid flippases enable precursor B cells to flee engulfment by macrophages. Proc Natl Acad Sci U S A. 2018;115(48):12212-12217.

18. Marsh B, Blelloch R. Single nuclei RNA-seq of mouse placental labyrinth development. Elife. 2020;9:e60266.

19. Peterson AC, et al. Parallel reaction monitoring for high resolution and high mass accuracy quantitative, targeted proteomics. Mol Cell Proteomics. 2012;11(11):1475-1488.

20. Baldridge RD, Graham TR. Two-gate mechanism for phospholipid selection and transport by type IV P-type ATPases. Proc Natl Acad Sci U S A. 2013;110(5):E358-E367.

21. Riekhof WR, Voelker DR. The yeast plasma membrane P4-ATPases are major transporters for lysophospholipids. Biochim Biophys Acta. 2009;1791(7):620-627.

22. Lee S, et al. Transport through recycling endosomes requires EHD1 recruitment by a phosphatidylserine translocase. EMBO J. 2015;34(5):669-688.

23. Nakanishi $\mathrm{H}$, et al. Transport cycle of plasma membrane flippase ATP11C by cryo-EM. Cell Rep. 2020;32(13):108208.

24. Kay JG, et al. Phosphatidylserine dynamics in cellular membranes. Mol Biol Cell. 2012;23(11):2198-2212.

25. Li G, et al. Efficient replacement of plasma membrane outer leaflet phospholipids and sphingolipids in cells with exogenous lipids. Proc Natl Acad Sci U S A. 2016;113(49):14025-14030.

26. Rong $X$, et al. ER phospholipid composition modulates lipogenesis during feeding and in obesity. JClin Invest. 2017;127(10):3640-3651.

27. Chen Y, Cao Y. The sphingomyelin synthase family: proteins, diseases, and inhibitors. Biol Chem. 2017;398(12):1319-1325.

28. Kiyokawa E, et al. Spatial and functional heterogeneity of sphingolipid-rich membrane domains. J Biol Chem. 2005;280(25):24072-24084.

29. Zager RA, et al. Sphingomyelinase and membrane sphingomyelin content: determinants ofProximal tubule cell susceptibility to injury. JAm Soc Nephrol. 2000;11(5):894-902.

30. Infante RE, Radhakrishnan A. Continuous transport of a small fraction of plasma membrane cholesterol to endoplasmic reticulum regulates total cellular cholesterol. Elife. 2017;6:e25466.

31. Maekawa M. Domain 4 (D4) of Perfringolysin O to visualize cholesterol in cellular membranes-the update. Sensors (Basel). 2017;17(3):504-514.

32. Sugiura $Y$, et al. Visualization of the cell-selective distribution of PUFA-containing phosphatidylcholines in mouse brain by imaging mass spectrometry. J Lipid Res. 2009;50(9):1776-1788.

33. Boycott KM, et al. Rare-disease genetics in the era of next-generation sequencing: discovery to translation. Nat Rev Genet. 2013;14(10):681-691.

34. Devaux PF. Static and dynamic lipid asymmetry in cell membranes. Biochemistry.
1991;30(5):1163-1173.

35. Zachowski A. Phospholipids in animal eukaryotic membranes: transverse asymmetry and movement. Biochem J. 1993;294(pt 1):1-14.

36. Fujimoto K, et al. Transmembrane phospholipid distribution revealed by freeze-fracture replica labeling. JCell Sci. 1996;109(pt 10):2453-2460.

37. Timcenko M, et al. Structure and autoregulation of a P4-ATPase lipid flippase. Nature. 2019;571(7765):366-370.

38. Naito T, et al. Phospholipid flippase ATP10A translocates phosphatidylcholine and is involved in plasma membrane dynamics. J Biol Chem. 2015;290(24):15004-15017.

39. Lipp NF, et al. Lipid exchangers: cellular functions and mechanistic links with phosphoinositide metabolism. Front Cell Dev Biol. 2020;8:663.

40. Holthuis JCM, Menon AK. Lipid landscapes and pipelines in membrane homeostasis. Nature. 2014;510(7503):48-57.

41. Lee JM, et al. A nuclear-receptor-dependent phosphatidylcholine pathway with antidiabetic effects. Nature. 2011;474(7352):506-510.

42. Musille PM, et al. Phospholipid - driven gene regulation. FEBS Lett. 2013;587(8):1238-1246

43. Mühle C, et al. Characterization of acid sphingomyelinase activity in human cerebrospinal fluid. PLoS One. 2013;8(5):e62912.

44. Takahashi I, et al. Distribution of acid sphingomyelinase in human various body fluids. Tohoku J Exp Med. 2000;192(1):61-66.

45. Kornhuber J, et al. Secretory sphingomyelinase in health and disease. Biol Chem. 2015;396(6-7):707-736.

46. Naito $\mathrm{T}$, et al. Movement of accessible plasma membrane cholesterol by the GRAMD1 lipid transfer protein complex. Elife. 2019;8:e51401.

47. Li C, et al. Crosstalk between acid sphingomyelinase and inflammasome signaling and their emerging roles in tissue injury and fibrosis. Front Cell Dev Biol. 2019;7:378.

48. Wang K, et al. ANNOVAR: functional annotation of genetic variants from high-throughput sequencing data. Nucleic Acids Res. 2010;38(16):e164.

49. Tokuhiro K, et al. Protein disulfide isomerase homolog PDILT is required for quality control of sperm membrane protein ADAM3 and male fertility. Proc Nat Acad Sci U S A. 2012;109(10):3850-3855

50. Nozawa K, et al. Sperm-borne phospholipase C zeta-1 ensures monospermic fertilization in mice. Sci Rep. 2018;8(1):1315-1310

51. Morita S, et al. Plat-E: an efficient and stable system for transient packaging of retroviruses. Gene Ther. 2000;7(12):1063-1066. 
52. Higuchi R. Recombinant PCR. In PCR Protocols: A Guide To Methods and Applications. Innis MA, et al, eds. Academic Press;1990:177-188.

53. Watanabe-Fukunaga R, et al. Lymphoproliferation disorder in mice explained by defects in Fas antigen that mediates apoptosis. Nature. 1992;356(6367):314-317

54. Palacios R, Steinmetz M. Il-3-dependent mouse clones that express B-220 surface antigen, contain Ig genes in germ-line configuration, and generate B lymphocytes in vivo. Cell.1985;41(3):727-734.
55. Shimada Y, et al. The C-terminal domain of perfringolysin $\mathrm{O}$ is an essential cholesterol-binding unit targeting to cholesterol-rich microdomains. Eur J Biochem. 2002;269(24):6195-6203.

56. Shevchenko A, et al. In-gel digestion for mass spectrometric characterization of proteins and proteomes. Nat Protoc. 2006;1(6):2856-2860.

57. Miller BT, et al. CHARMMing: a new, flexible web portal for CHARMM. J Chem Inf Model. 2008;48(9):1920-1929.

58. Jo S, et al. CHARMM-GUI: a web-based graphical user interface for CHARMM. J Comput Chem. 2008;29(11):1859-1865.

59. Phillips JC, et al. Scalable molecular dynamics with NAMD. J Comput Chem. 2005;26(16):1781-1802.

60. Ryckaert JP, et al. Numerical integration of the cartesian equations of motion of a system with constraints: molecular dynamics of n-alkanes. J Comput Phys. 1977;23(3):327-341.

61. Feller SE, et al. Constant pressure molecular dynamics simulation: the Langevin piston method. JChem Phys. 1998;103(11):4613-4621. 